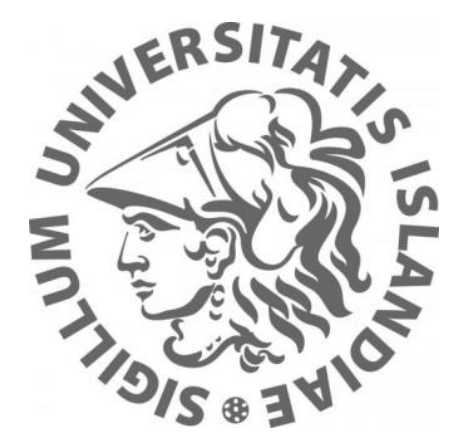

\title{
Gesture Interpretation Control System Using Convolutional Neural Networks
}

Benedikt Jón Baldursson

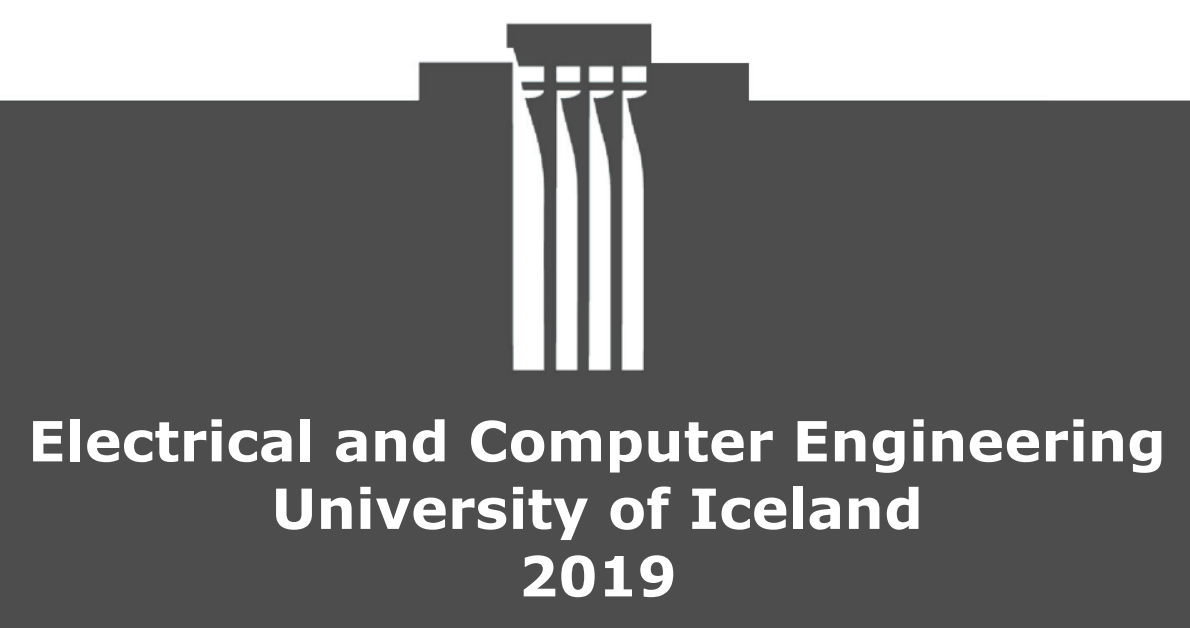





\title{
Gesture Interpretation Control System Using Convolutional Neural Networks
}

\author{
Benedikt Jón Baldursson
}

20 ECTS thesis submitted in partial fulfilment of a Baccalaureus Scientiarum degree in Mechatronic Engineering

\author{
Advisors \\ Behnood Rasti \\ Karl Sölvi Guðmundsson
}

Faculty of Electrical and Computer Engineering

School of Engineering and Natural Science

University of Iceland

Reykjavík, December 2019 
Gesture Interpretation Control System Using Convolutional Neural Networks 20 ECTS thesis submitted in partial fulfilment of a Baccalaureus Scientarium degree in Mechatronics Engineering Technology

Copyright @ 2019 Benedikt Jón Baldursson

All rights reserved

Faculty of Electrical and Computer Engineering

Engineering and Natural Sciences

University of Iceland

Skólabraut 3

220 Hafnarfjörður

Phone: 5784000

Bibliographic Information:

Benedikt Jón Baldursson, 2019, Gesture Interpretation Control System Using

Convolutional Neural Networks, BSc thesis, School of Engineering and Natural Sciences, University of Iceland, pp. 38.

Printing: Háskólaprent ehf., Fálkagata 2, 107 Reykjavík

Reykjavík, December 2019 


\section{Abstract}

This thesis proposes a non-invasive control system for electrical wheelchairs utilizing facial gestures of individuals captured by a real-time monocular camera. The images are interpreted with a convolutional neural network that achieves up to $99.6 \%$ overall accuracy. The control system uses an embedded system with a graphics processing unit for predicting real-time throughput with fast classification time. This solution offers excellent versatility, where the user can make a gesture to depict a command of his choice.

Keywords-non-invasive, gestures, real-time, convolutional neural network, machine learning.

\section{Útdráttur}

Pessi ritgerð leggur fram inngriplausan stjórnbúnað fyrir rafmagnshjólastóla sem nemur andlitshreyfingar einstaklinga sem fangaðar eru með eineygis myndavél. Myndirnar eru síðan túlkaðar með feðmingar-djúptauganeti sem nær að 99.6\% nákvæmni. Stjórnkerfið notast við ígreypt kerfi með skjákorti til að greina myndir í rauntíma með skjótum rökleiðslutíma. Lausnin býður upp á mikla fjölbreyttni í notkun par sem notandi notar andlitslátbragð til að lýsa skipun að eigin vali. 

To the love of my life Pórdís Dröfn who has supported me throughout this study. 



\section{Table of Contents}

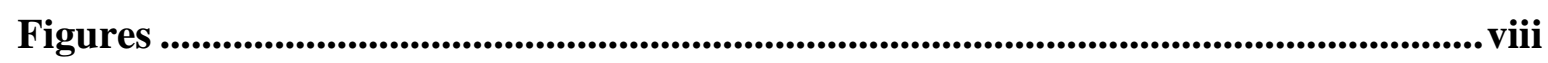

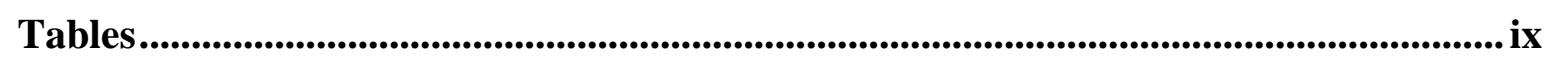

Acknowledgements .......................................................................................................................... xi

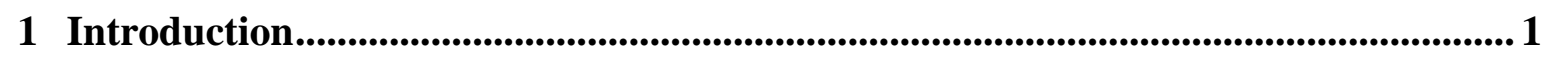

1.1 Common Electrical Wheelchair Controllers ..................................................... 1

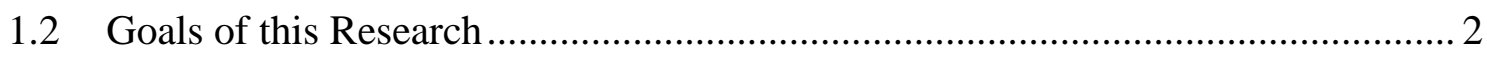

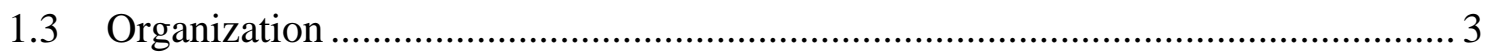

2 State of the Art .............................................................................................................. 5

$2.1 \quad$ Smart Wheelchairs …………………………………………………….

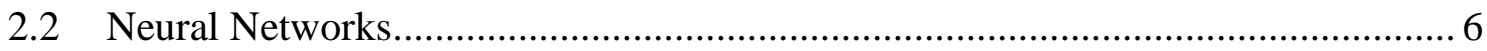

3 Requirement Analysis................................................................................................................9

4 Implementation and Methods.................................................................................................. 11

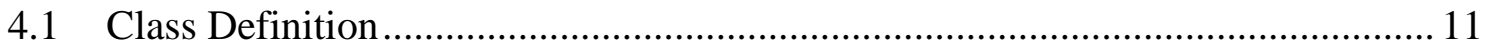

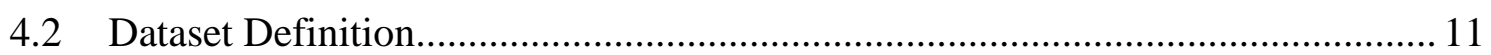

4.3 Dataset Pre-processing .................................................................................. 13

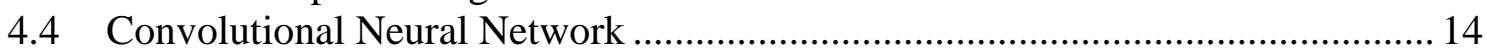

4.5 Interfacing of the Control System ..................................................................... 20

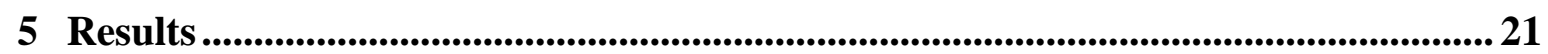

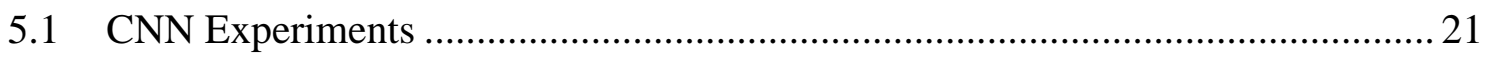

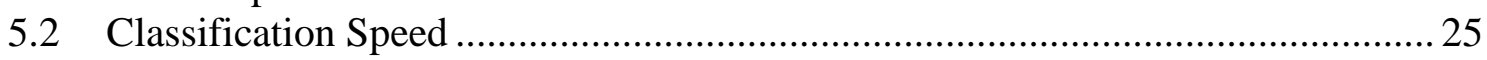

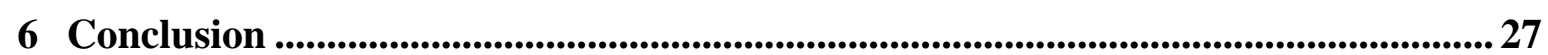

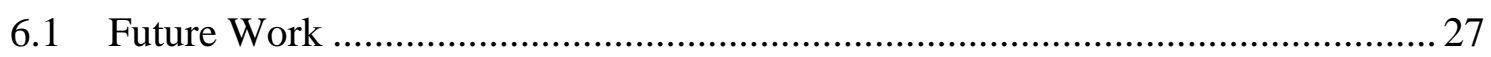

References............................................................................................................................. 29

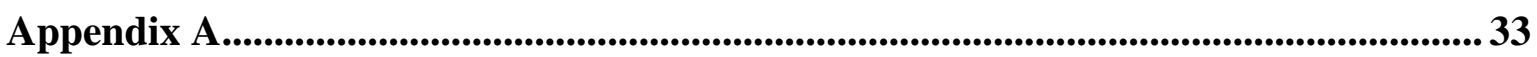

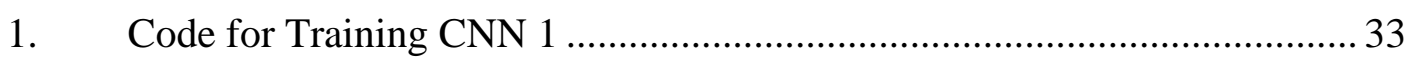

2. Keras to TensorRT Conversion Code .......................................................... 35

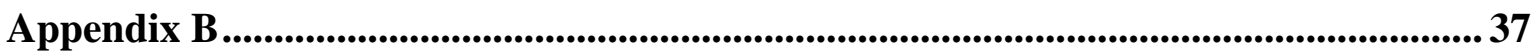

1. Sample of Expression Variance in Dataset ................................................... 37

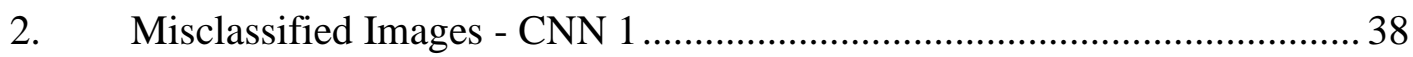

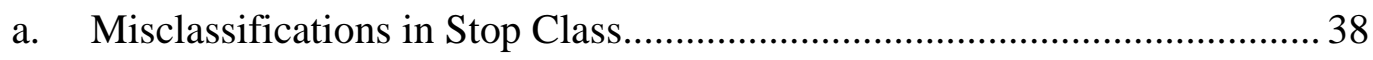

b. Misclassifications in Left Class ................................................................... 38 


\section{Figures}

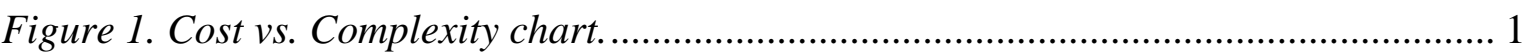

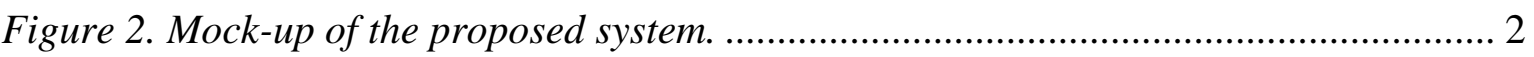

Figure 3. Difference in occlusion mixing, and lighting conditions.................................. 12

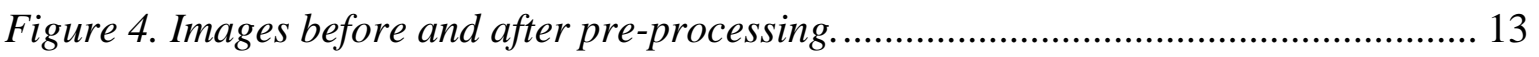

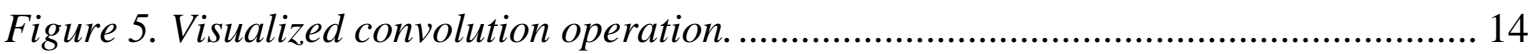

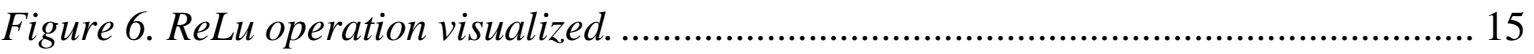

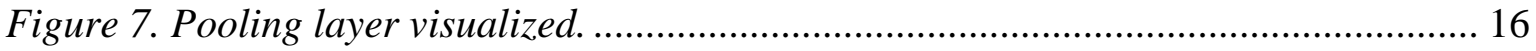

Figure 8. Input feature map flattened. ........................................................................ 16

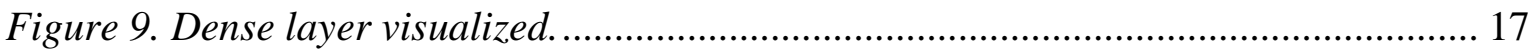

Figure 10. Neurons temporarily dropped randomly ..................................................... 17

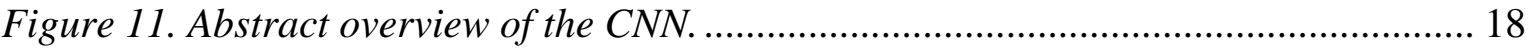

Figure 12. Components of the learning pattern box. .................................................... 18

Figure 13. Components of the weight tuning box. ......................................................... 19

Figure 14. The flowchart proposed for the control system............................................ 20

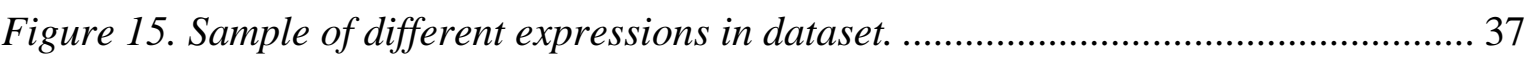

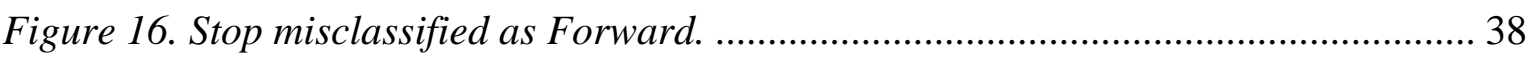

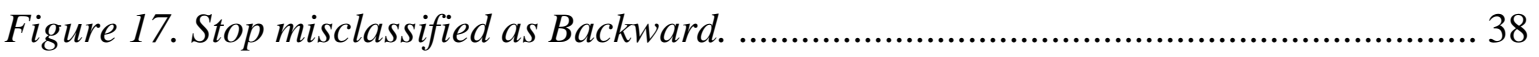

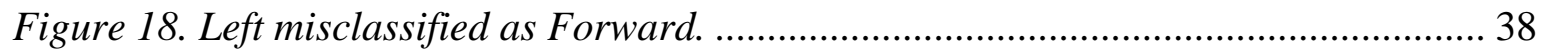




\section{Tables}

Table 1. Comparison between embedded systems......................................................... 9

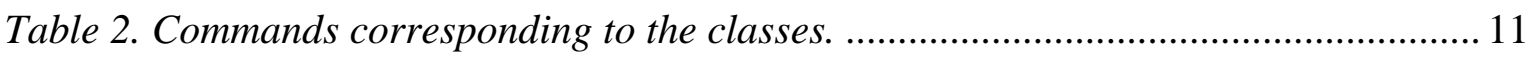

Table 3. The confusion matrix for CNN 1 on the test samples......................................... 22

Table 4. The confusion matrix for CNN 2 on the test samples. ......................................... 22

Table 5. Only 32 output filters

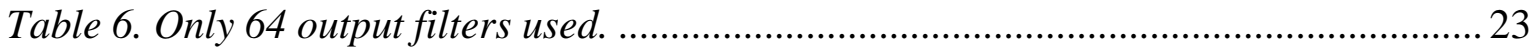

Table 7. First convolutional layer with 32 output filters................................................. 24

Table 8. First convolutional layer with 64 output filters.................................................... 24

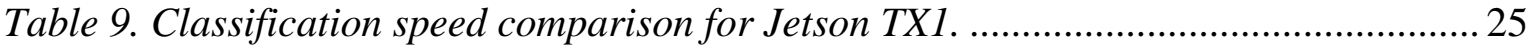





\section{Acknowledgements}

I want to express my gratitude and appreciation to my instructor Behnood Rasti, as well as Karl S. Guðmundsson who gave me the excellent opportunity to do this project and also present it at the Biomedical Innovations and Applications IEEE conference, that experience has been invaluable to me and enabled me to finalize this thesis.

Secondly, I would also like to thank my parents, Baldur and María, and my fiancée, Pórdís, that have supported me throughout my studies. I could not have done this without them. 



\section{Introduction}

Current solutions for people with tetraplegia are invasive and insufficient for any form of mobility. Independence in moving about without caretakers help is impossible. This can decrease the quality of the life of an impaired person.

\subsection{Common Electrical Wheelchair Controllers}

The most frequent used controller is the joystick controller (JC). However, it is not suitable for every electrical wheelchair user. The most common controller for people with tetraplegia is the sip-and-puff switch ( $\mathrm{SnP})$. The function of the sip-and-puff switch requires the user to suck and blow for executing essential functions. Therefore, it limits the number of commands to perform. A relatively new method for disabled people is implanting a magnetic stud into the tongue of the user. This allows the tongue muscle to be used as a joystick for controlling the wheelchair. This is called Tongue-Drive System (TDS)[1]. A Cost vs. Complexity graph can be seen in Figure 1 below. The figure shows only the most common controllers used and where the proposed system, Gesture Interpretation (GI) system, is relative to these conventional control systems.

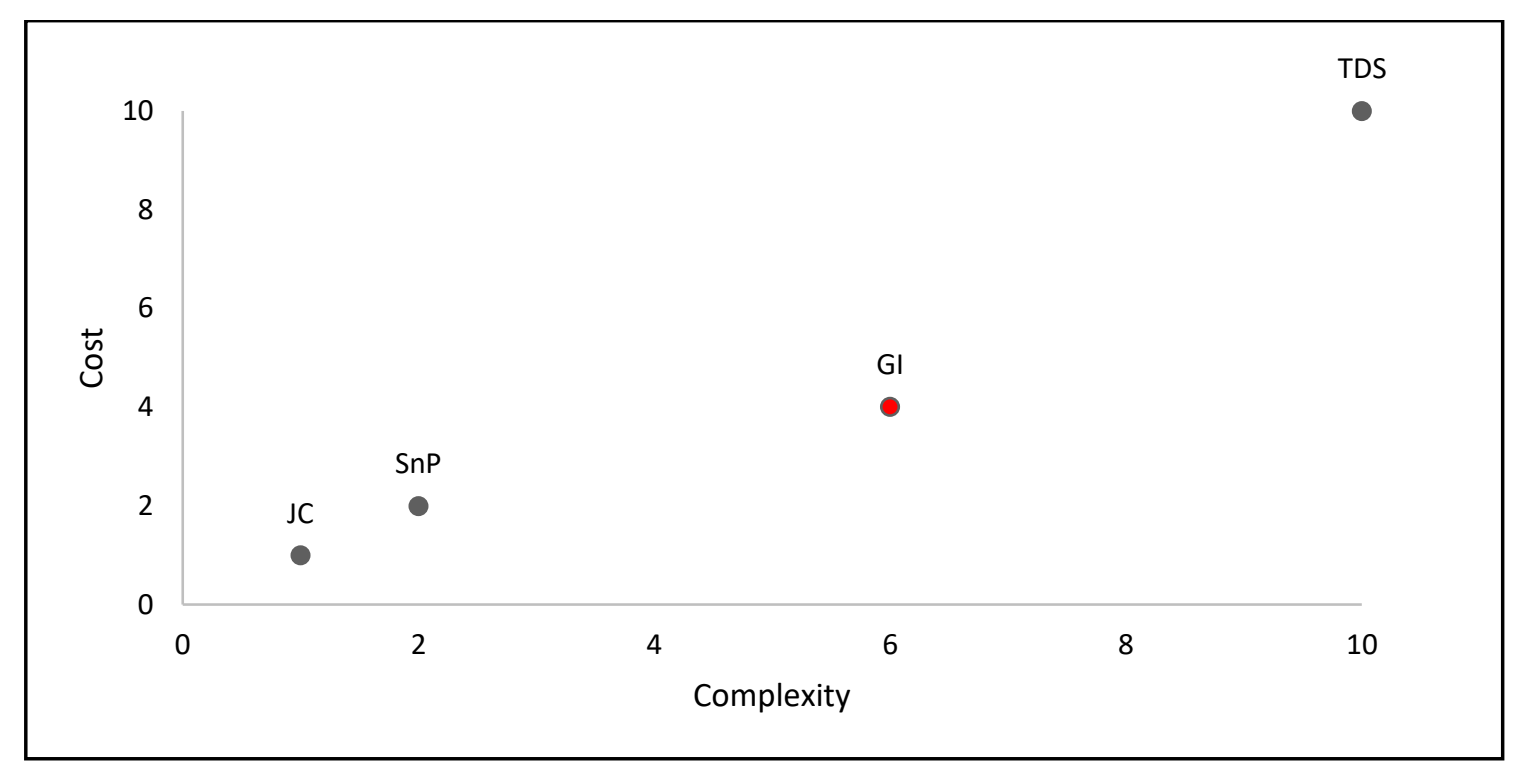

Figure 1. Cost vs. Complexity chart.

The joystick controller is the most inexpensive and not complicated in setting up. The sipand-puff switch is also inexpensive but more complex in implementation than the joystick controller. The Tongue-Drive System requires invasive surgery and is complex to set up, making it an expensive system. The proposed Gesture Interpretation system is relatively complex to implement and relatively inexpensive. 
There is a need to improve the mobility of tetraplegic people to increase their independence and quality of life. A device or system which makes it possible for an individual to move from one room to another, going to the park or the grocery store independently is needed. This can be accomplished with a Gesture Interpretation control system. The Gesture Interpretation control system proposed in this thesis is a system comprising an imaging device and artificial intelligence using a combination of deep learning convolutional neural network $(\mathrm{CNN})$ and obstacle avoidance algorithms.

\subsection{Goals of this Research}

The goal of this research work is to develop a non-invasive control system for mobile devices. The system monitors real-time gesture images from the user. Subsequently, the system translates the gesture images into commands using a CNN. The proposed solution offers the flexibility to adapt data to the gestures of any individual.

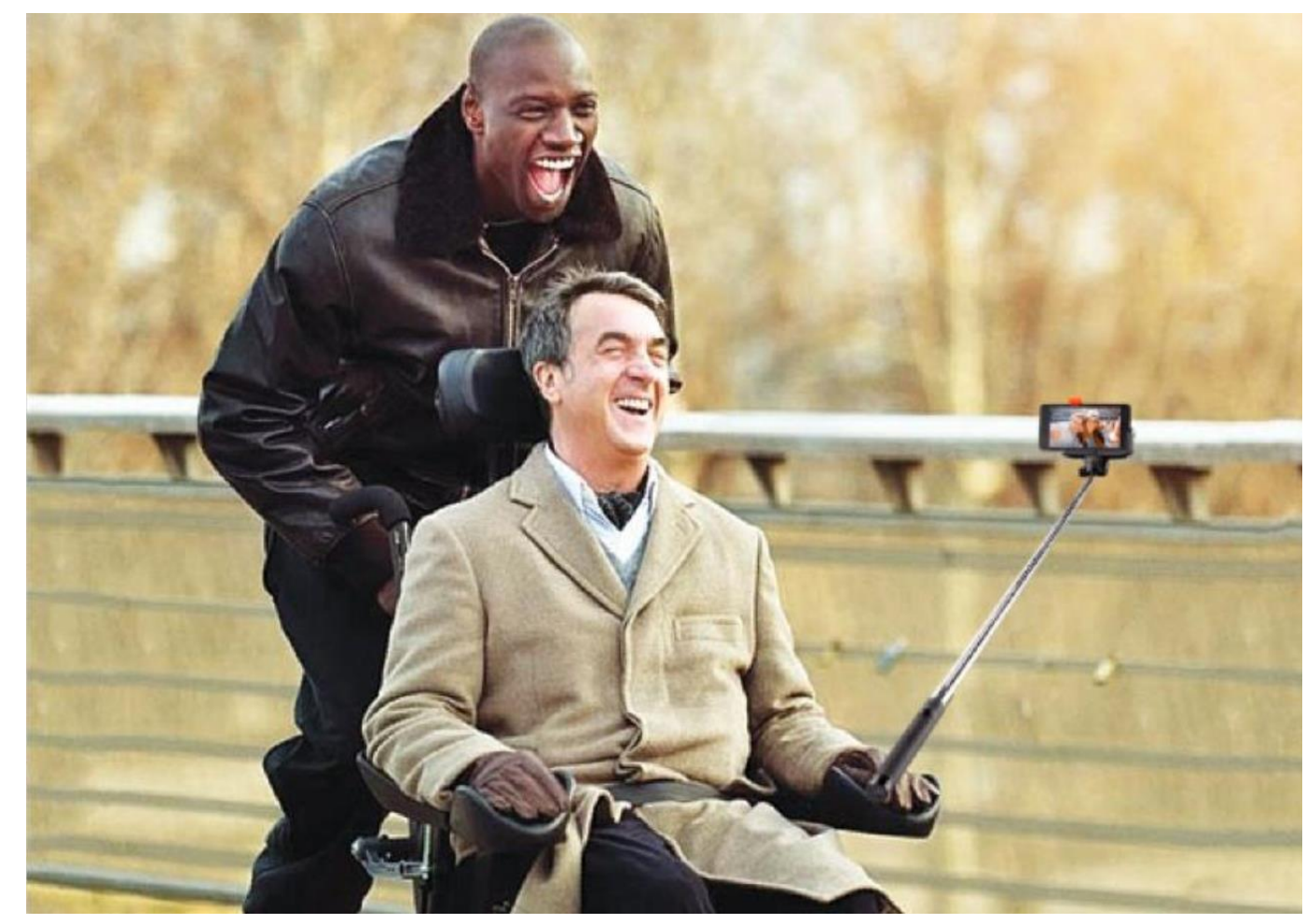

Figure 2. Mock-up of the proposed system.

This thesis reports on the results of a student's final project in BSc in Mechatronic Engineering at the University of Iceland. The project is limited to 5 basic commands using Jetson TX1 Development Kit ${ }^{\mathrm{TM}}$ from NVIDIA and Keras ${ }^{\mathrm{TM}}$ with TensorFlow ${ }^{\mathrm{TM}}$ backend for the CNN. 


\subsection{Organization}

The thesis is structured as follows: Chapter 2 describes the state of the art. Chapter 3 shows the requirement analysis. Chapter 4 discusses in detail the method and implementation developed in this research. Chapter 5 presents the results of the experiments. Finally, chapter 6 concludes this thesis and gives directions for future work. 



\section{State of the Art}

Most of the standard control mechanisms are limited by their input data allowing only a few commands. The first wheelchair designed in 1986 for people with impaired mobility was a self-navigating wheelchair that uses an onboard microcomputer, camera, and an ultrasonic sensor [2]. The chair was limited by the environment. Another novel design was the NavChair ${ }^{\text {TM }}$ [3] assistive wheelchair navigation system designed in 1999. NavChair ${ }^{\mathrm{TM}}$ uses algorithms for general obstacle avoidance, automatic wall following, and door passages.

Eye tracking methods or the absolute point of gaze (POG) methods are used in many wheelchair designs for physically challenged people. The three dominant and widely used eye tracking methods are video-oculography (VOG), pupil-cornea reflection (PCR), and electro-oculography (EOG). VOG methods use video cameras as data input that is stored in and interpreted by a computer. These systems can be head-mounted or put on a wheelchair. PCR method gathers highly accurate data from the user's gaze. PCR systems utilize artificial infrared (IR) light source. The PCR method is widely used in the scientific domain. EOG solution offers high temporal accuracy in measuring relative movements of the eyes. Two electrodes are situated around the orbit of the eye that can be measured. The EOG method requires wearable goggles that attach to the skin around the eyes [4].

\subsection{Smart Wheelchairs}

M. A. Eid et al. proposed a system in "A Novel Eye-Gaze-Controlled Wheelchair System for Navigating Unknown Environments: Case Study with a Person with ALS" [5] that enables people with limited movement to control a wheelchair with eye gaze in unknown environments. The control system of their proposed method allows the user to move around. The system uses 3D geometry data of the ambient environment collected by a 3D camera. The geometric data is then articulated with an algorithm that identifies obstacles, plans routes, and navigates through unknown environments. The system uses an adaptable N-cell grid-based graphical user interface (GUI). The calibration method used for the eye tracking algorithm is minimal, making setup for new users simple. A participant with ALS (amyotrophic lateral sclerosis) was involved in a case study, showing that this system is feasible as a controller.

In "A multimodal wheelchair control system based on EEG signals and Eye tracking fusion" [6], F. B. Taher et al. propose a control system based on a fusion of electroencephalography (EEG) and eye tracking techniques. The eye tracking technique uses a webcam for detecting the gaze of both eyes. Viola and Jones proposed an algorithm in "Robust Real-Time Face Detection" [7] for extracting regions of each eye individually. For face detection in the proposed control system, F. B. Taher et al. use the algorithm presented by Viola and Jones. After face detection and isolation of the eyes, an adaptive threshold algorithm locates the pupils. The location of the pupils is stationed in a $3 \times 3$ matrix that depicts direction. EEG

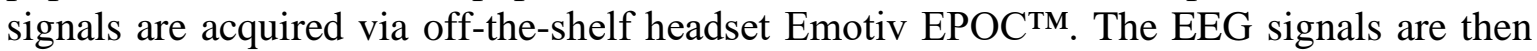
interpreted by a computer into commands. The system is tested on control performance by 
eye tracking, EEG signals, and EEG/eye tracking fusion. F. B. Taher et al. show that using the EEG signals as the central controller is the most accurate, but it is too power consuming. Therefore, the fusion of eye tracking and EEG is proposed.

In their research, "Head Gesture Controlled Wheelchair for Quadriplegic Patients" [8], Machangpa and Chingtham designed a robotic wheelchair that uses head gestures as commands. The obstacle avoidance control system is used in conjunction with the head gesture control system. Cost-effective accelerometer and gyroscope sensors are used for head gesture monitoring. The sensors measure pitch, roll, and yaw data. Movement commands are acquired from this data. For obstacle avoidance, an ultrasonic sensor is used to detect obstacles. The system is centred on a raspberry pi computer for data processing and controlling the wheelchair.

\subsection{Neural Networks}

T. Chan et al. [9] proposed PCANet, a very simple deep learning network. This network can be used as a simple baseline for image classification. It is based on block-wise histograms, binary hashing, and cascaded principal component analysis (PCA). Multistage filter banks are learnt firstly by using the PCA method. These PCA convolutional filters reduce dimensionality, which significantly speeds up feature learning. For the indexing and pooling, a simple block histogram and binary hashing are used. Benchmarking on visual datasets for different tasks shows that the proposed network is on par with state-of-the-art networks.

There are four crucial steps in face recognition process: Detect, align, represent, and classify. In [10], alignment and representation are at the forefront. The location of the face is fixed at the pixel level after alignment. The faces are 3D modelled on a generic 3D model face. Modelling uses 67 fiducial points, from the aligned image, for deriving the 3D facial representation. The 3D model data is then passed into a deep neural network (DNN). This method is highly accurate on large datasets, $97.5 \%$ accuracy on the LFW dataset. However, it takes 0.23 seconds to classify one image.

A Deep learning based face recognition attendance system using $\mathrm{CNN}$ is proposed by $\mathrm{M}$. Arsenovic et al. [11]. The CNN has a few essential steps, which are highly advanced techniques: CNN cascade for detecting a face and generating facial embedding with a CNN. A small dataset is used, CNNs usually require big datasets to achieve good results. Image augmentation is proposed, which generates artificial noise and occlusions to enlarge the dataset. The first step of the proposed algorithm is detecting a face using the CNN cascade method proposed by H. Li et al. [12]. In the second step, M. Arsenovic et al. use 68 facial landmarks for alignment. Thirdly, the face is embedded onto Euclidean space for measuring face similarities. The network is trained with a support vector machine (SVM) classifier. The proposed system achieves over $95 \%$ accuracy.

Detection of facial landmarks is a big part of pre-processing the data for more accurate training with DNNs. In [13], two types of deep convolutional neural networks (DCNNs) are used in conjunction. Combining these two DCNNs shows good results. One type detects components constrained on facial landmarks. The other type detects components constrained on the facial contour. The former DCNN branches out to higher layers by capturing features of intricate local facial landmarks (eyes, eyebrows, nose, nose-bridge, and mouth) and 
treating it as a regression problem. The latter DCNN exploits the relationship between facial landmarks and facial contour landmarks. This relationship is used due to difficulty in the detection of the pose, subject appearance, and background noise. The two DCNN are separately trained, which improves the detection of components in the facial landmarks.

Different poses, illumination, expression, age, and occlusion create significant problems in face recognition tasks. Y. Sun et al. propose a system for solving that problem by reducing intrapersonal variations while enlarging interpersonal differences [14]. Using deep learning and utilizing signals of face identification and verification as supervision for feature representation. Identification classifies input images into identity classes. Verification classifies pairs of images that belong to the same identity or not (i.e., binary classification). Carefully designed DCNNs are used for learning the deep identification-verification features (DeepID2). The network, proposed by Y. Sun et al., achieves an accuracy of $99.15 \%$ on LFW dataset for face verification.

Y. Sun et al. [15] use a high-performance deep convolutional network (DeepID2+) for face recognition. Using the supervisory signal identification-verification method, presented in [14] by Y. Sun et al. The difference lies in more dimensions of hidden representations for convolutional layers. The training dataset is enlarged, from 160.000 to 290.000 images. Supervision to first convolutional layers is added with identification-verification supervisory signals. DeepID2+ achieves $99.47 \%$ accuracy on the LFW dataset. 



\section{Requirement Analysis}

A motorized wheelchair, under consideration, can travel at speed $(s)$ of approximately 1.8 $\mathrm{m} / \mathrm{s}$. Therefore, the control system must have enough processing power to respond quickly and smoothly to a change in user command. The onboard camera module is the limiting factor of the proposed system. The camera is capable of grabbing frames at 30 frames per second $(F R)$. To estimate the approximate granularity $(g)$ of travel per frame, we use Equation (1).

$$
g=\frac{s}{F R}
$$

Therefore, the granularity of travel per frame is calculated by $g=1.8 / 30=0.06 \mathrm{~m}$. This gives us a precision of $6 \mathrm{~cm}$ of distance travelled between frames at the maximum speed of wheelchairs.

As a result, the control system requires computational power to acquire and process at least 30 frames per second.

For the present project, the NVIDIA ${ }^{\mathrm{TM}}$ Jetson TX1 development board [16] is available for use. However, it is essential for the completeness of the project to evaluate the processing requirements and compare a few different available systems from NVIDIA in Table 1.

Table 1. Comparison between embedded systems.

\begin{tabular}{|c|c|c|c|}
\hline Specifications & Jetson NANO & Jetson TX1 & Jetson TX2 \\
\hline GPU & 128 -core Maxwell & 256 -core Maxwell & $256-$ cores Pascal \\
\hline CPU & $4 \times 1.43 \mathrm{GHz}$ & $4 \times 1.73 \mathrm{GHz}$ & $4 \times 2 \mathrm{GHz}+2 \times 2 \mathrm{GHz}$ \\
\hline Memory & $4 \mathrm{~GB}$ & $4 \mathrm{~GB}$ & $8 \mathrm{~GB}$ \\
\hline Storage & MicroSD & $16 \mathrm{~GB}$ eMMC & $32 \mathrm{~GB}$ eMMC \\
\hline Performance & $472 \mathrm{GFLOPS}$ & 1 TFLOPS & $1.3 \mathrm{TFLOPS}$ \\
\hline Power & $5-10 \mathrm{~W}$ & $10-15 \mathrm{~W}$ & $7.5 \mathrm{~W}$ \\
\hline Power Supply & $5 \mathrm{~V}$ DC & $5.5-19.6 \mathrm{~V}$ DC & $5.5-19.6 \mathrm{~V}$ DC \\
\hline Size & $69.6 \mathrm{~mm} \times 45 \mathrm{~mm}$ & $87 \mathrm{~mm} \times 50 \mathrm{~mm}$ & $87 \mathrm{~mm} \times 50 \mathrm{~mm}$ \\
\hline Price & $\$ 99.00$ & $\$ 259.00$ & $\$ 399.00$ \\
\hline
\end{tabular}


The camera accompanying the TX1 development board captures 640 x 480 x 10-bit monochromatic images. Assuming the same camera module is available and applicable for the three versions of the Jetson family given in Table 1, we can compare which module would be the most cost-effective option.

All of the embedded systems in Table 1 are suitable for fast classification time, due to the GPU integration. However, Jetson TX1 is used to implement this project since that was available. 


\section{Implementation and Methods}

\subsection{Class Definition}

As mention before, any individual can utilize this solution. The flexibility of the system allows the user to choose any set of gestures, with distinction in mind. The dataset created contains five classes for five basic commands; forward, backward, stop, right, and left. Gestures chosen for the classes in this project are shown in Table 2.

Table 2. Commands corresponding to the classes.

\begin{tabular}{|l|l|}
\hline Command & Gesture \\
\hline Go forward (FWD) & Tilt head forward \\
\hline Go backward (BWD) & Tilt head backward \\
\hline Stop & Close eyes \\
\hline Go right & Turn head clockwise \\
\hline Go left & Turn head anti-clockwise \\
\hline
\end{tabular}

The control system is not limited to this set of gestures. This set is chosen because it is descriptive, distinct, and appropriate for proof of concept (POC) project.

\subsection{Dataset Definition}

The dataset used in this research has images of one subject. Each class has approximately 500 images; 250 images for training, 35 images for validation, and 215 images for testing. Images are captured with a webcam. The dataset has few occlusions present, three different lighting conditions, and variable facial expressions. Occlusions presented in images are a hat, glasses, hood, and a mix of those objects. Variance in the mixing of occlusion and lighting is shown in Figure 3. 


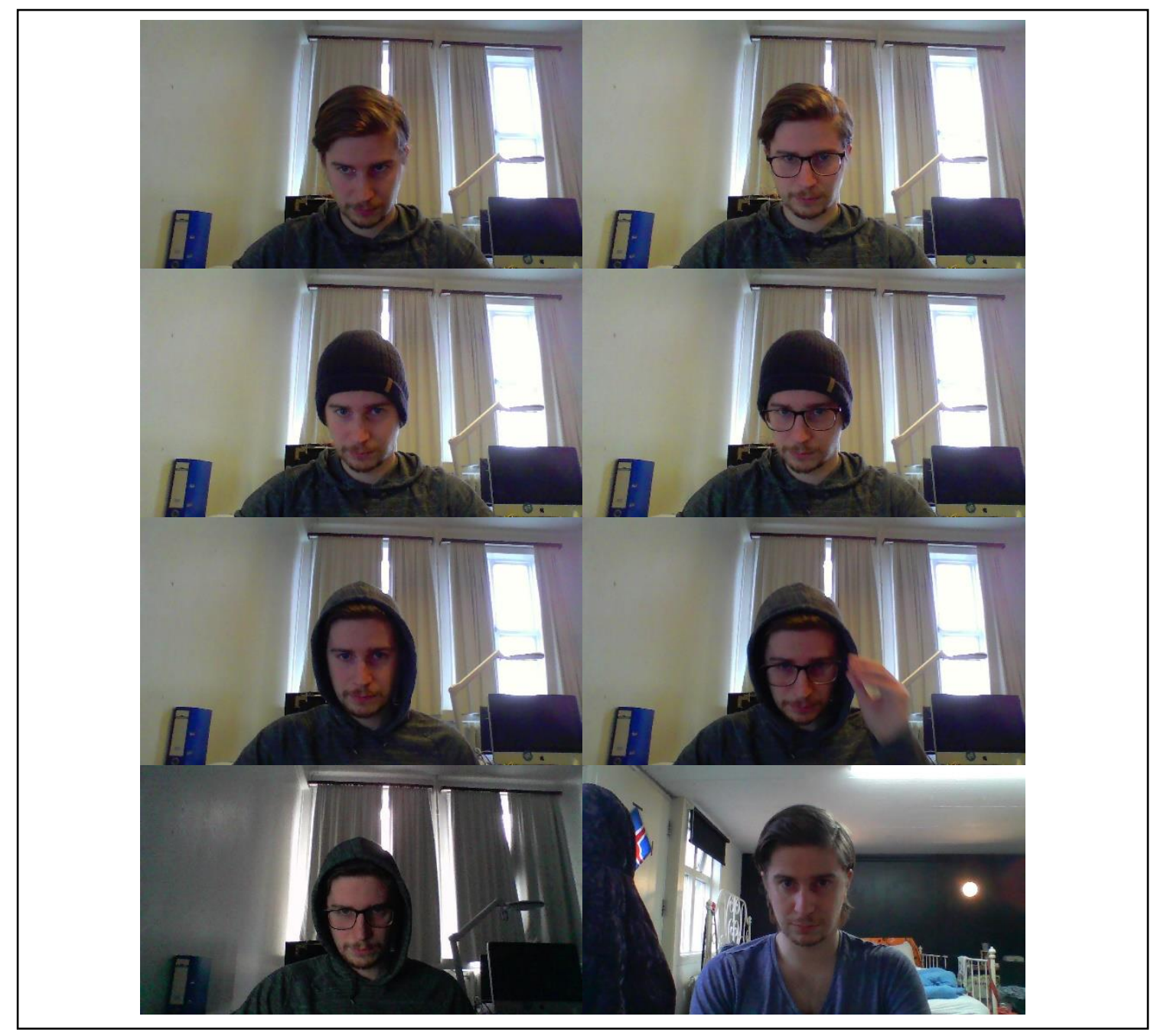

Figure 3. Difference in occlusion mixing, and lighting conditions.

The objects used for occlusion purposes were worn in an orderly fashion. The following three steps are used for describing how the wearable items were used. Step 1: No wearable item used, then glasses added. Step 1 is shown in the first row of images in Figure 3. Step 2: Hat is worn, then glasses added. Step 2 is shown in the second row of images in Figure 3. Step 3: Hood put up, then glasses added. Step 3 is shown in the third row of images in Figure 3. The images are taken while steps 1-3 are carried out. While taking the images, various random expressions were made. Appendix B shows samples of expressions used. The lighting conditions were changed after Steps 1-3 were finished, then Steps 1-3 were repeated for the next lighting conditions. The first lighting conditions can be seen in row 1-3 in Figure 3. The second and third lighting conditions can be seen in row 4 in Figure 3. 


\subsection{Dataset Pre-processing}

The dataset is pre-processed using the method proposed in "Joint Face Detection and Alignment Using Multitask Cascaded Convolutional Networks“ [18] by Zhang et al., called Multitask Cascaded Neural Network (MTCNN) method. The MTCNN method detects facial landmarks and crops the face out of the picture, minimizing background noise. The code used resizes images to $182 \times 182$ pixels with 24 -bit depth. Figure 4 compares the raw and preprocessed images.

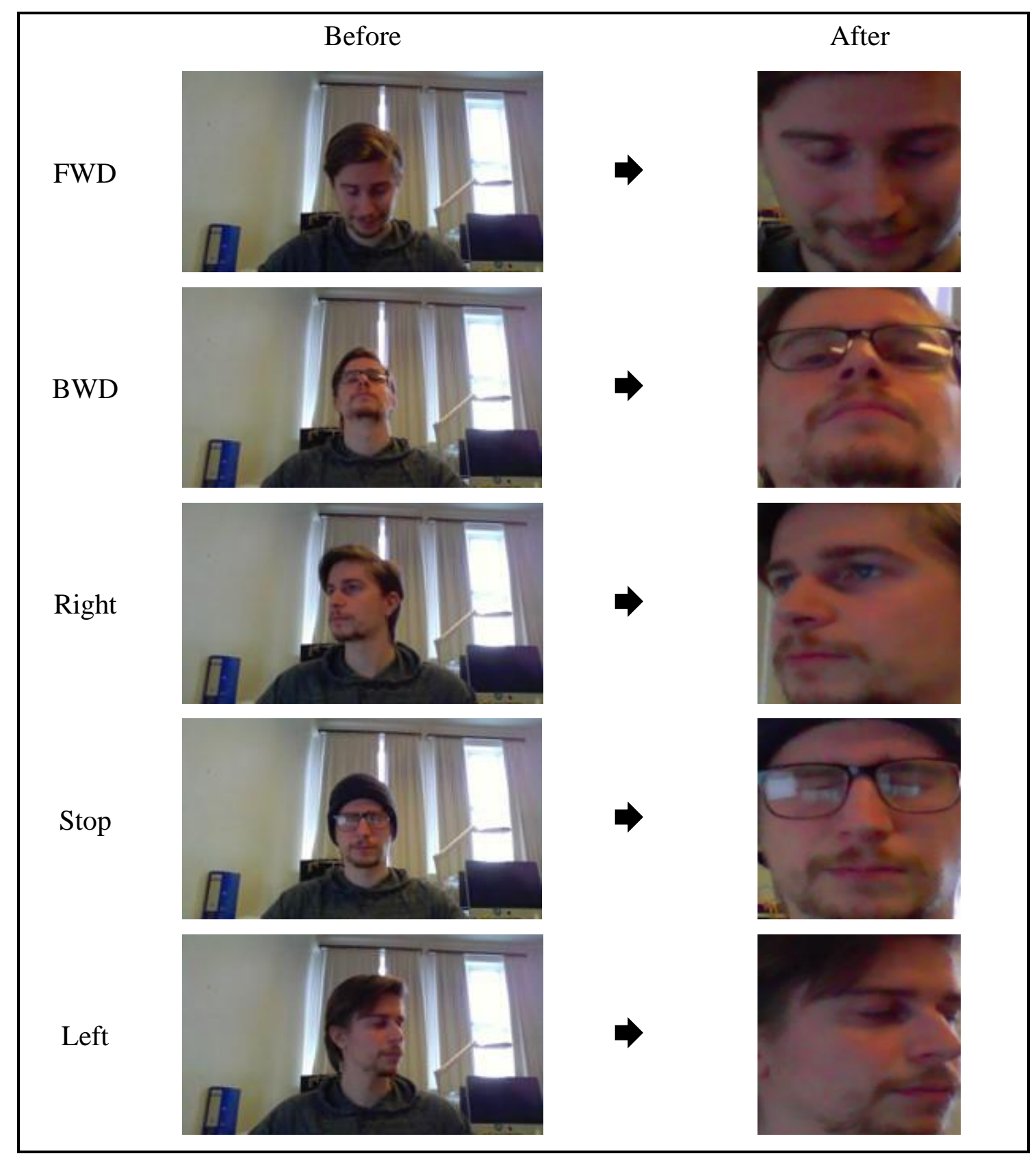

Figure 4. Images before and after pre-processing. 


\subsection{Convolutional Neural Network}

The CNNs are implemented with Keras ${ }^{\mathrm{TM}}$ API with TensorFlow ${ }^{\mathrm{TM}}$ backend. The CNNs train on data using the SGD (Stochastic Gradient Descent) optimizer. The SGD optimizer reduces computation needed allowing faster training. By single randomly picked examples, each iteration estimates the gradient instead of computing the gradient, explained by Bottou in "Stochastic Gradient Descent Tricks" [18].

The first layer in the proposed algorithm is a two-dimensional convolution (Conv2D). This layer uses convolutional methods on the input image. When an image has passed through the first layer, the image is regarded as an intermediate feature map. The feature map is then passed on to the next layer. This method is a linear filtering operation which produces a tensor of outputs by doing spatial convolution on the input image with a created convolution kernel given by Equation (2).

$$
g(x, y)=f(x, y) \otimes h(x, y)=\sum_{\tau_{1}} \sum_{\tau_{2}} f\left(\tau_{1}, \tau_{2}\right) h\left(x-\tau_{1}, y-\tau_{2}\right)
$$

Where $f$ is the input image or feature map, $h$ is the convolution kernel, and $g$ is the output feature map of the convolution operation. The convolution kernel, $h$, slides over the input image, $f$. At each location, matrix multiplication is performed and summed up into the output feature map, $g$. Figure 5 shows the visualized representation of the convolutional operation.

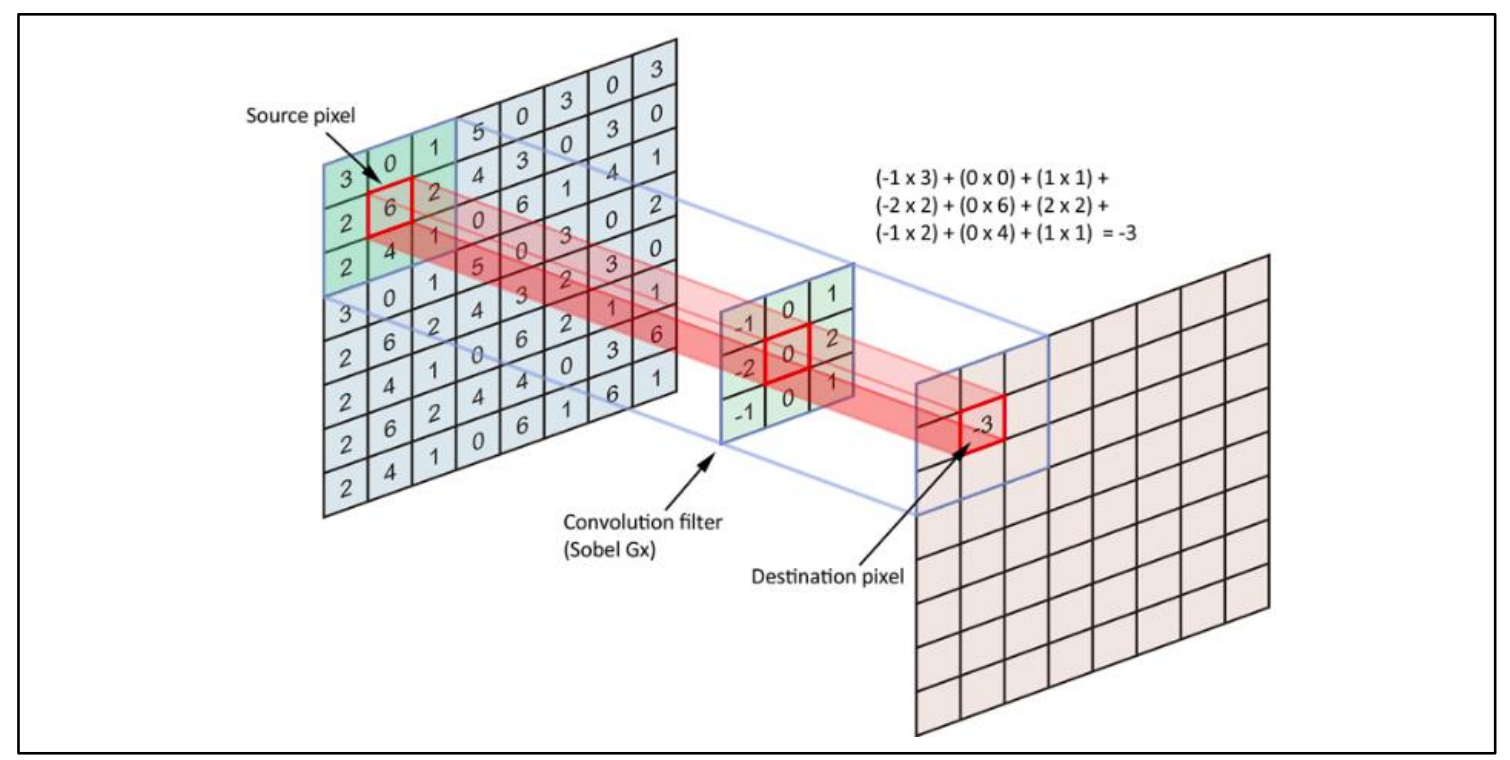

Figure 5. Visualized convolution operation [19].

The spatial dimension of the input image or feature map can decrease, depending on the padding and stride hyperparameters. Padding is applied to keep the spatial dimension constant. Stride signifies the steps taken by the filter over the input image or feature map. Another significant hyperparameter is the filter output. The filter output is the number of patterns that the convolutional layer outputs. These hyperparameters are handcrafted while the weights that are hidden within the layer change (i.e., adjust) when training on images. 
The weights are essential for solving target problems such as classifying images. The visualized representation of the convolution operation in Figure 5 uses; 1x1 padding, stride 1 , input size $8 \times 8 \times 1$ with kernel size $3 \times 3 \times 1$, and output filter size $8 \times 8 \times 1$. The input feature map spatial dimension is $10 \times 10 \times 1$ when padding has been applied. Therefore, the spatial dimension for the output feature map is $8 \times 8 \times 1$.

The second layer, Rectified Linear unit (ReLu), is a non-linear activation function. The function sets all negative values to zero with no change in spatial or depth information of the input data. That allows cheaper computation and faster convergence. The ReLu function can be described with Equation (3).

$$
y=\max (0, x)
$$

The input feature map, $x$, is passed through function $\max ($ ), which returns values less than zero as zero. The ReLu operation is visualized in Figure 6.

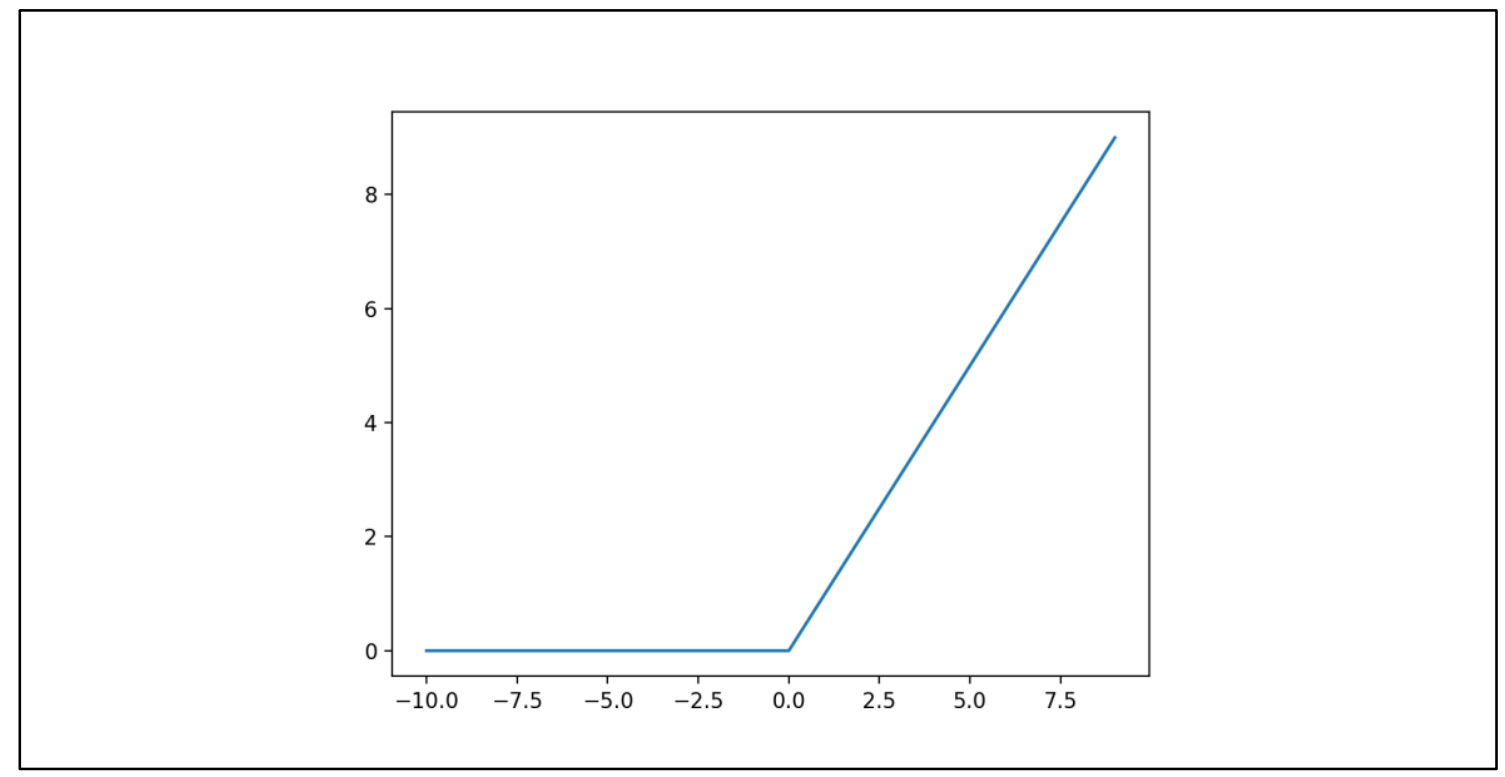

Figure 6. ReLu operation visualized [20].

Figure 6 shows a line plot of ReLu operation for negative and positive inputs. The slope is the function derivative. Negative inputs have slope 0 , and positive inputs have slope 1 . The linear behaviour of the ReLu operation output makes the network easier to optimize.

The third layer is max pooling (MaxPooling2D). This operation reduces the spatial information and the number of parameters, which decreases the computational cost. It subsamples the input feature map, so little spatial information is lost when performing this operation. Figure 7 shows how the max pooling function decreases the spatial size of the input feature map. 


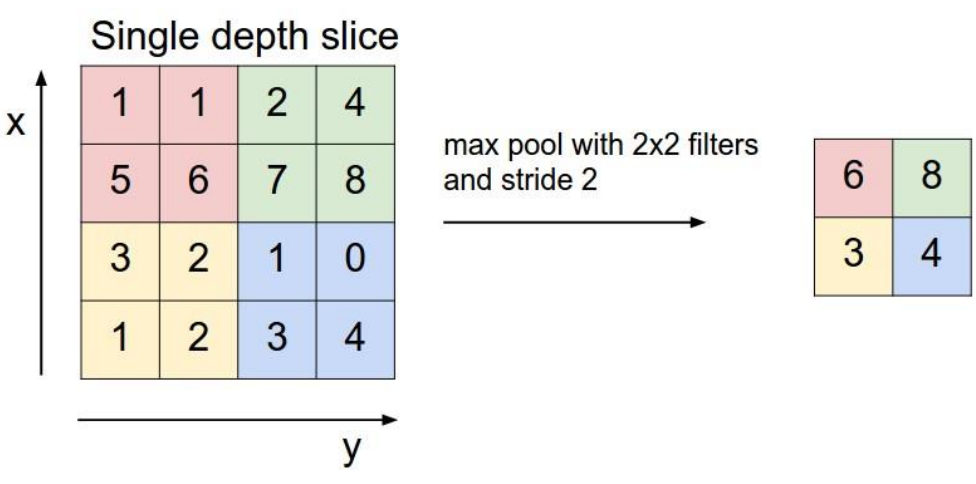

Figure 7. Pooling layer visualized [21].

The input feature map of size $4 \times 4$ is decreased to size $2 \times 2$ by using $2 \times 2$ filter and stride 2 , seen in Figure 7.

The next layer (called Flatten) flattens the input feature map into a 1D vector for passing into a fully connected layer. Figure 8 depicts the flattening operation.

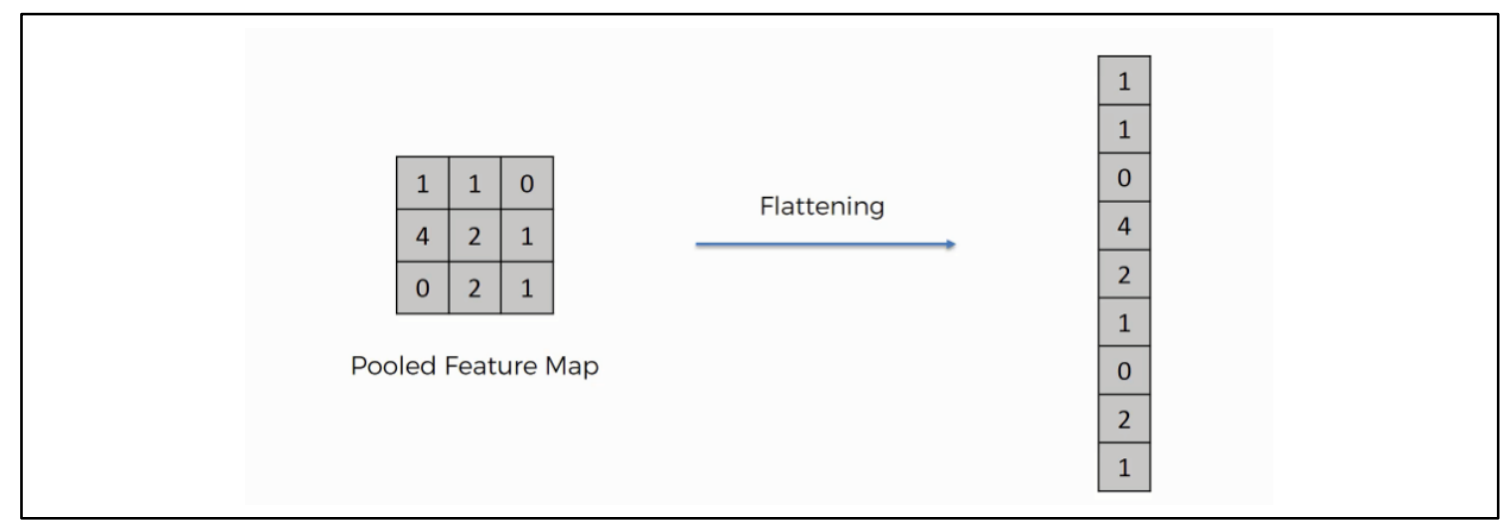

Figure 8. Input feature map flattened [22].

The flattening operation takes in input feature map of size $3 \times 3$ and returns a $1 \times 9$ feature map, seen in Figure 8.

The fully connected layer is called the Dense layer. This layer connects every neuron with each neuron from the previous layer. These neurons are trainable and get updated during backpropagation. The Dense layer has one hyperparameter, the number of units (i.e., neurons). Figure 9 shows how the neurons all connect to each neuron in the previous layer. 


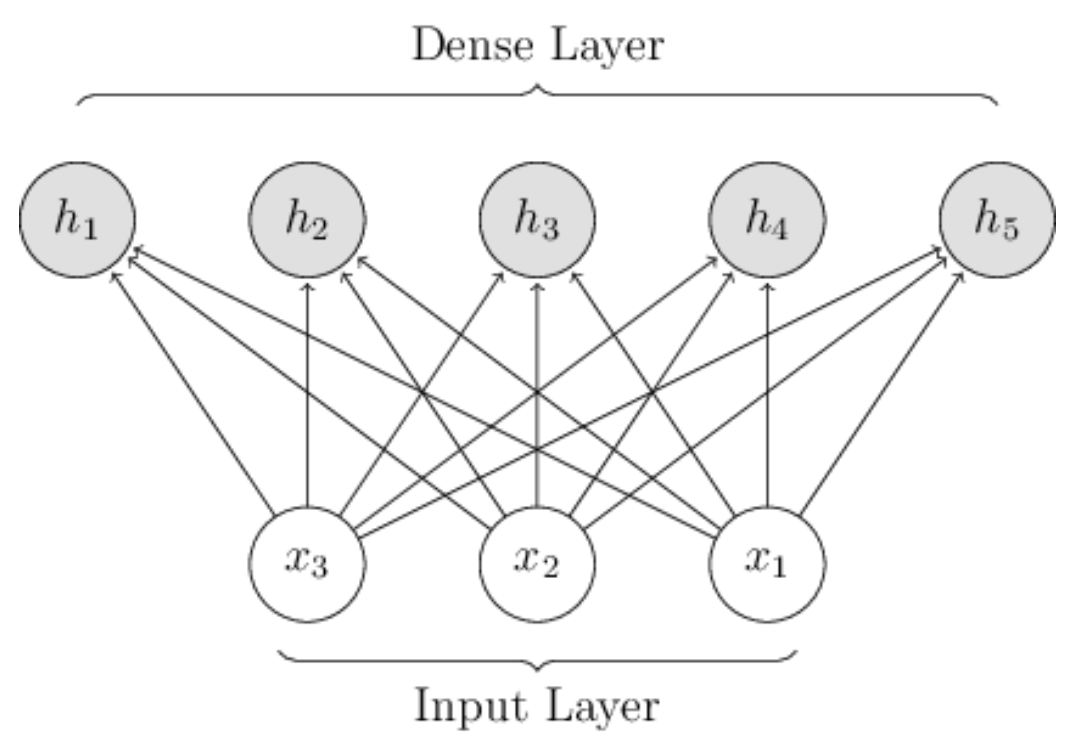

Figure 9. Dense layer visualized [23].

The number of units, $h$, is set to 5 in Figure 9. These units are considered as neurons in machine learning applications. The number of units in the last Dense layer in a neural network must be set to the corresponding number of classes.

The next layer (Dropout layer) temporarily removes inputs to a layer which prevents overfitting. Units are randomly dropped along with their connections during the training of neural networks proposed by Srivastava et al. in "Dropout: A Simple Way to Prevent Neural Networks from Overfitting" [24]. The Dropout layer has one hyperparameter, the fraction of units dropped. The Dropout layer is visualized in Figure 10.

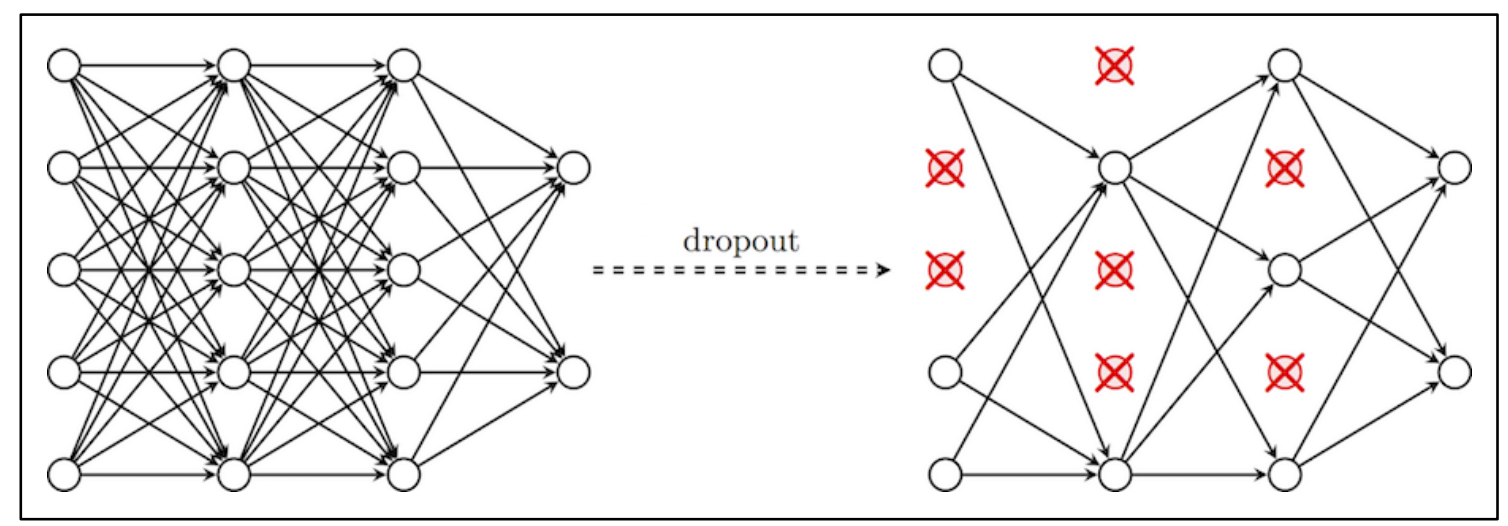

Figure 10. Neurons temporarily dropped randomly [24].

Figure 10 shows how the neurons and their connections are temporarily dropped. In column $1,40 \%$ of the units are dropped. In column $2,60 \%$ of the units are dropped. In column 3 , $40 \%$ of the units are dropped. Units are dropped randomly. Therefore, the units dropped in Figure 10 will not be the same for the next image. 
The final layer (SoftMax layer) is an activation function that takes in tensor input and applies SoftMax normalization. The function outputs the probability distribution for each class. The SoftMax function can be described by Equation (4).

$$
S\left(\theta_{i}\right)=\frac{e^{\theta_{i}}}{\sum_{j} e^{\theta_{j}}}
$$

The sum of Equation (4) is always 1, where $S\left(\theta_{i}\right)$ is the score for each class for a given input image.

The building blocks of the CNNs are explained in Figures 11-13. Figure 11 illustrates a highlevel abstraction of the CNNs.

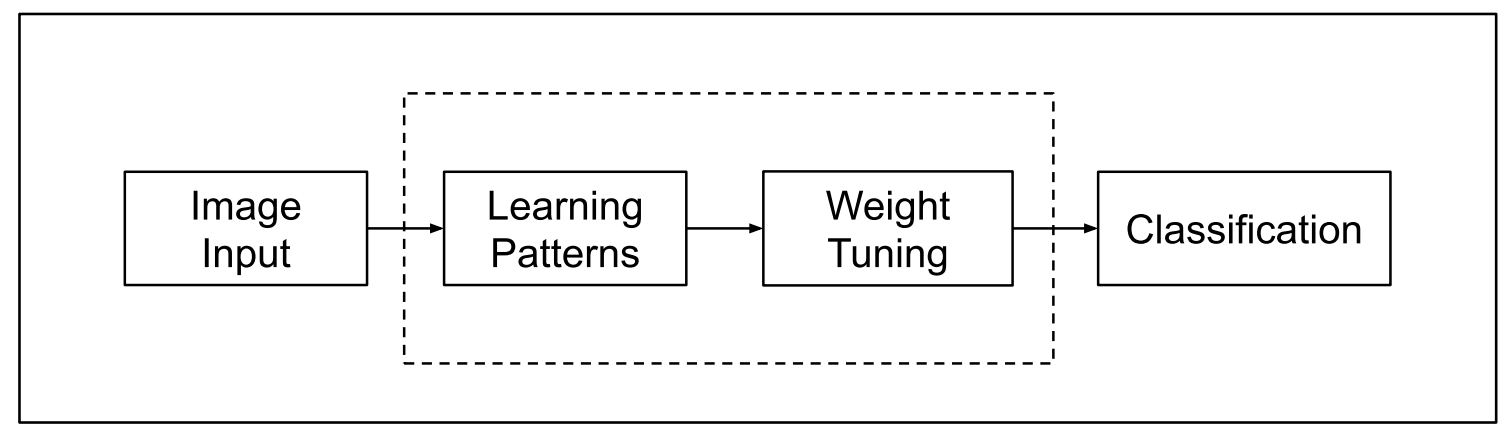

Figure 11. Abstract overview of the CNN.

First, image is received, then patterns are learnt (Learning Patterns) from the input image. That is followed by tuning the weights of the neurons (Weight Tuning), then classification accuracy is acquired. The classification is distributed accordingly for each class, depending on the image anomalies. Figure 12 shows the layers used in the Learning Patterns box.

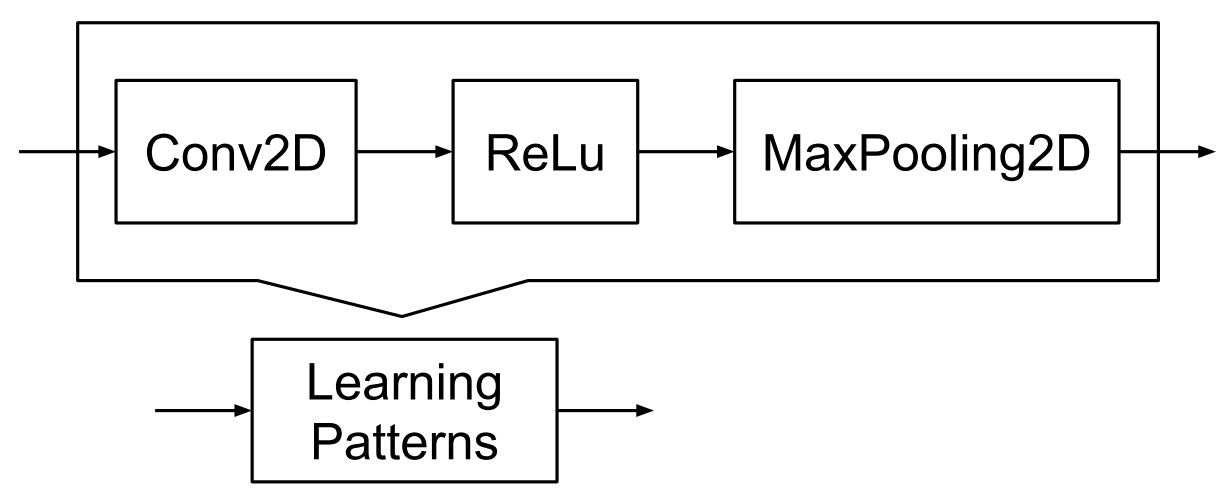

Figure 12. Components of the learning pattern box. 
The convolution, ReLu, and max pooling operations are performed inside the Learning Patterns box. Here specific patterns are learnt from the input image or feature map. The spatial dimension of the input image or feature map is decreased, which consequently decreases the number of parameters. This box can be repeated for experimental purposes in getting different results in classification accuracy. The output filter hyperparameter of the convolution operation can also be changed for different results.

The rest of the operations used in the CNNs are inside the Weight Tuning box, Figure 13. This box fine-tunes neurons in the network. The classification probabilities are also acquired from this box.

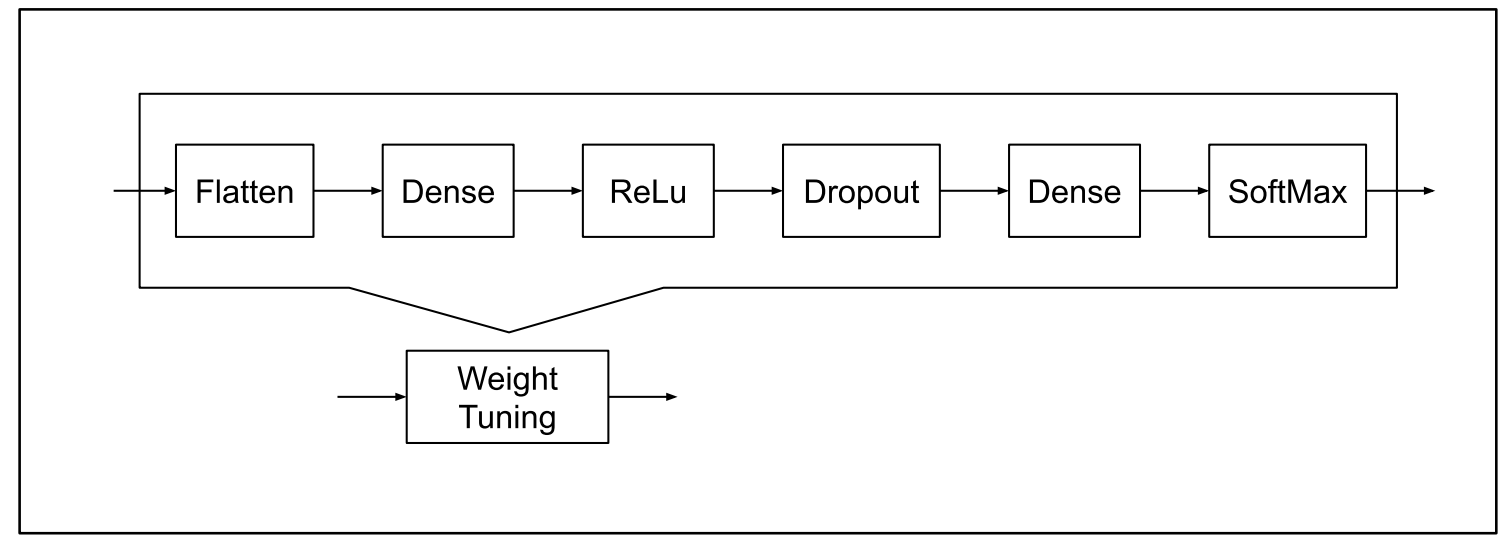

Figure 13. Components of the weight tuning box.

The operations that are performed in the Weight Tuning box are Flatten, Dense, ReLu, Dropout, and SoftMax. This box is not repeated. The hyperparameters for Dense and Dropout layers can be changed, which consequently changes the number of parameters. The last Dense layer has to be set to the corresponding number of classes. 


\subsection{Interfacing of the Control System}

The General-Purpose Input and Output (GPIO) of the Jetson TX1 will be used for this project for outputting commands to a DC (direct current) motor controller. The onboard camera module on the Jetson TX1 will be used for the video input.

Figure 14 depicts the flowchart of the control system. First, a frame is captured from video input. Then, the frame is processed by cropping face. Classification is then done on the cropped face. If classification accuracy is above $99 \%$, then the command is executed, and the next frame is grabbed. If classification accuracy is less than $99 \%$, then no command is executed, and the next frame is grabbed.

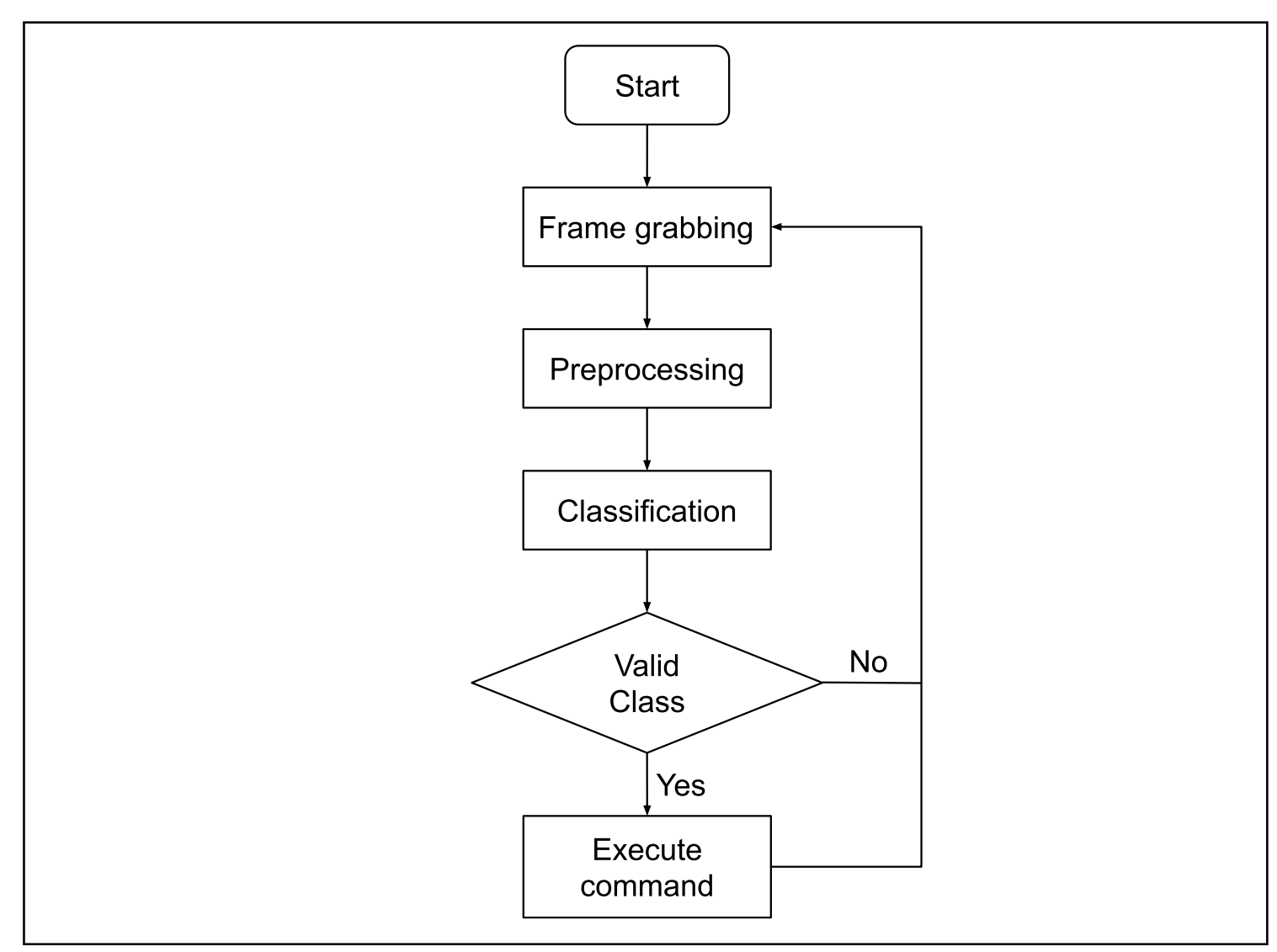

Figure 14. The flowchart proposed for the control system. 


\section{Results}

\subsection{CNN Experiments}

In this thesis, we experiment with several different CNNs. First, two different CNNs are tested, called CNN 1 and CNN 2. The difference is in the hyperparameters for convolution, Dense, and Dropout layers. There is also a difference in the number of layers used for these two CNNs. The rest of CNN experimentations are implemented with CNN 1 as the base model. CNN 1 and CNN 2 are explained in detail below.

\section{Experimental setup for CNN 1.}

In this experiment, 3 convolutional layers are used. The number of epochs is set to 50, and the batch size is set to 32 . The first and second Conv2D layers have 32 output filters that use kernel size $3 \times 3$ with stride 1 along height and width, the third Conv2D layer has 64 output filters that use kernel size $3 \times 3$ with stride 1 along height and width. ReLu is followed by each Conv2D layer and the first Dense layer. The MaxPooling2D operations have size 2 in both spatial dimensions. The first Dense layer has 64 in dimensionality of the output space. The second Dense layer has 5 in dimensionality of the output space, corresponding to the output classes. The Dropout layer drops 0.5 fractions of the input units. SoftMax is the last layer, and the total number of layers in CNN 1 is 15 .

\section{Experimental setup for CNN 2.}

Here 4 convolutional layers are used. The number of epochs is set to 50, and the batch size is set to 32. The first and second Conv2D layers have 32 output filters that use kernel size $3 \times 3$ with stride 1 along height and width. The third and fourth Conv2D layers have 64 output filters that use kernel size $3 \times 3$ with stride 1 along height and width. ReLu is followed by each Conv2D layer and the first Dense layer. The MaxPooling2D operations have size 2 in both spatial dimensions. The first Dense layer has 256 in dimensionality of the output space. The second Dense layer has 5 in dimensionality of the output space, corresponding to the output classes. The first and second Dropout layer drops 0.25 fractions of the input units, and the third Dropout layer drops 0.5 fractions of the input units. SoftMax is the last layer, and the total number of layers in CNN 2 is 18.

A PC is used to speed up the training process. The PC used has 8GB 2432 CUDA core GPU (GTX1070Ti), 4 core CPU (Intel i7-2600), and DDR3 32GB 1600MHz RAM with Ubuntu 18.04LTS. Training CNN 1 on the dataset takes 7 minutes and 38 seconds, with 50 epochs, processing 154 images per second. Training for CNN 2 on the dataset takes 7 minutes and 45 seconds, with 50 epochs, processing 154 images per second. Trained models are then transferred to the development board for doing classification.

The confusion matrices obtained from the test images for CNN 1 and CNN 2 are given, respectively, in Tables 3 and 4. As can be seen in Table 3, CNN 1 has 100\% accuracy in 
Forward, Backward, and Right classes. In the Stop class one sample is misclassified as Right and two samples misclassified as Backward. In the Left class, one sample is misclassified as Forward. The overall accuracy for CNN 1 is $99.6 \%$, and the average accuracy (the average over the accuracies of the five classes) is $99.7 \%$.

Table 3. The confusion matrix for CNN 1 on the test samples.

\begin{tabular}{|c|c|c|c|c|c|c|}
\hline Class & FWD & BWD & Right & Stop & Left & Accuracy \\
\hline FWD & $\mathbf{2 0 7}$ & 0 & 0 & 0 & 0 & $\mathbf{1}$ \\
\hline BWD & 0 & $\mathbf{2 0 5}$ & 0 & 0 & 0 & $\mathbf{1}$ \\
\hline Right & 0 & 0 & $\mathbf{1 9 7}$ & 0 & 0 & $\mathbf{1}$ \\
\hline Stop & 1 & 2 & 0 & $\mathbf{2 2 9}$ & 0 & $\sim \mathbf{0 . 9 8 7 1}$ \\
\hline Left & 1 & 0 & 0 & 0 & $\mathbf{2 8 1}$ & $\mathbf{\sim 0 . 9 9 6 5}$ \\
\hline
\end{tabular}

CNN 2 has 100\% accuracy in Forward and Backward classes. In the Right class, three samples are misclassified as Forward, four samples as Backwards, and two samples as Stop. In the Stop class, six samples are misclassified as Forward and three samples as Backwards. In the Left class, only two samples are misclassified as Backwards. The overall accuracy for CNN 2 is $98.3 \%$, and the average accuracy is $98.2 \%$. CNN 1 has higher accuracies than CNN 2 in all classes, and CNN 1 is also smaller with fewer parameters. Therefore, CNN 1 is the more applicable network for the proposed control system in terms of speed and accuracy.

Table 4. The confusion matrix for CNN 2 on the test samples.

\begin{tabular}{|c|c|c|c|c|c|c|}
\hline Class & FWD & BWD & Right & Stop & Left & Accuracy \\
\hline FWD & $\mathbf{2 0 7}$ & 0 & 0 & 0 & 0 & 1 \\
\hline BWD & 0 & $\mathbf{2 0 5}$ & 0 & 0 & 0 & $\mathbf{1}$ \\
\hline Right & 3 & 4 & $\mathbf{1 8 8}$ & 2 & 0 & $\sim \mathbf{0 . 9 5 4 3}$ \\
\hline Stop & 6 & 3 & 0 & $\mathbf{2 2 3}$ & 0 & $\sim \mathbf{0 . 9 6 1 2}$ \\
\hline Left & 0 & 2 & 0 & 0 & $\mathbf{2 8 0}$ & $\mathbf{\sim 0 . 9 9 2 9}$ \\
\hline
\end{tabular}

More experiments were implemented with CNN 1 as a base model. The experiments were done with the difference in repetitions of the Learning Patterns box (convolution, ReLu, and max pooling operations) and output filters of the convolutional layers. Therefore, CNNs are explained in terms of the number of convolutional layers and their number of output filters. 
The epochs were set to 20 . Tables 5-8 show the overall accuracy of every CNN from these experiments. In Table 5, CNNs using only 32 output filters for convolution layers are shown.

Table 5. Only 32 output filters used.

\begin{tabular}{|c|c|c|}
\hline Convolutional Layers & Output Filters & Accuracy \\
\hline 1 & 32 & $\sim 99.1986 \%$ \\
\hline 2 & $32-32$ & $\sim 96.2600 \%$ \\
\hline 3 & $32-32-32$ & $\sim 96.4381 \%$ \\
\hline
\end{tabular}

Using only 32 output filters, we get over $99 \%$ overall accuracy with only 1 convolutional layer, which is the best result for a 1 layer CNN. Using 2 layers with 32 output filters, we get over $96 \%$ overall accuracy. Using 3 layers with 32 output filters, we get over $96 \%$ overall accuracy. With less 32 output filter layers, the better the accuracy gets. When fewer layers are used, we get more parameters in the network with the CNN setup in this project. That makes the network computationally more expensive. Therefore, we get faster classification speed with more layers. That is due to the max pooling operation. Which subsequently gives fewer connections in the Dense layer. Table 6 shows CNNs using only 64 output filters for each convolutional layer.

Table 6. Only 64 output filters used.

\begin{tabular}{|c|c|c|}
\hline Convolutional Layers & Output Filters & Accuracy \\
\hline 1 & 64 & $\sim 91.5405 \%$ \\
\hline 2 & $64-64$ & $\sim 92.9653 \%$ \\
\hline 3 & $64-64-64$ & $\sim 97.2395 \%$ \\
\hline
\end{tabular}

Using only 64 output filters, we get the best results when 3 layers are used, over $97 \%$ overall accuracy. Using 1 layer with 64 output filters, we get over $91 \%$ overall accuracy. Using 2 layers with 64 output filters, we get over $92 \%$ overall accuracy. The more convolutional layers using 64 output filters, the better the accuracy gets. Using 64 output filters means more parameters in the network, making it computationally more expensive. Therefore, using 32 output filters would be wanted. CNNs using combinations of 32 and 64 output filters with 32 output filters in first convolutional layer are shown in Table 7. 
Table 7. First convolutional layer with 32 output filters.

\begin{tabular}{|c|c|c|}
\hline Convolutional Layers & Output filters & Accuracy \\
\hline 2 & $32-64$ & $\sim 94.8353 \%$ \\
\hline 3 & $32-32-64$ & $\sim 99.6438 \%$ \\
\hline 3 & $32-64-32$ & $\sim 94.9243 \%$ \\
\hline 3 & $32-64-64$ & $\sim 96.7943 \%$ \\
\hline
\end{tabular}

The best results are gotten from using the 32-32-64 output filter combination, $299.6438 \%$ overall accuracy. Using 2 layers with the 32-64 output filter combination, we get over $94 \%$ accuracy. Using 3 layers with the 32-64-32 output filter combination, we get over 94\% overall accuracy. Using 3 layers with the 32-64-64 output filter combination, we get over $96 \%$ overall accuracy. Table 8 shows CNNs using combinations of 32 and 64 output filters with 64 output filters in the first convolutional layer.

Table 8. First convolutional layer with 64 output filters.

\begin{tabular}{|c|c|c|}
\hline Convolutional Layers & Output filters & Accuracy \\
\hline 2 & $64-32$ & $\sim 98.4862 \%$ \\
\hline 3 & $64-32-32$ & $\sim 96.0819 \%$ \\
\hline 3 & $64-32-64$ & $\sim 91.8967 \%$ \\
\hline 3 & $64-64-32$ & $\sim 97.5957 \%$ \\
\hline
\end{tabular}

Here we get the best results from using 2 layers with the 64-32 output filter combination, over $98 \%$ overall accuracy. This $64-32$ output filter combination is the best result for a 2 layer CNN. Using 3 layers with the 64-32-32 output filter combination, we get over $96 \%$ overall accuracy. Using 3 layers with the 64-32-64 output filter combination, we get over 91\% overall accuracy. Using 3 layers with the 64-64-32 output filter combination, we get over $97 \%$ overall accuracy.

Some classes had perfect accuracy in the experimental setup where CNN 1 is used as the base model. Classification for Forward and Backward classes are 100\% accurate. The networks only misclassify images in Right, Left, and Stop classes. Right, and Left classes are very similar classes. Stop class has the least anomalies in the images, with only change in the eye region. Therefore, making Right, Left, and Stop classes harder to classify. 


\subsection{Classification Speed}

The classification speed is tested with TensorRT models on the embedded system, Jetson TX1. Three CNN models were tested, chosen with the best accuracy and difference in the number of convolutional layers in mind. The networks chosen were; CNN with 1 layer using 32 output filters, CNN with 2 layers using the 64-32 output filter combination, and CNN with 3 layers using the 32-32-64 output filter combination. Additionally, a fourth CNN with 3 layers using the 32-32-32 output filter combination was chosen for testing. The fourth CNN is also tested because it has the fewest parameters, 922,949 parameters. That would make it the fastest CNN out of experiments. These models were trained using Keras. The models were then ported to the Jetson TX1. The converted TensorRT model could not be ported to the Jetson TX1. Therefore, the same Keras model had to be converted to a TensorRT model on the Jetson TX1 with the same code. That is due to different versions of packages installed on the PC and Jetson TX1. Then each TensorRT model was tested against the Keras model counterpart yielding the same classification results. The frames per second for each model are shown in Table 9.

Table 9. Classification speed comparison for Jetson TX1.

\begin{tabular}{|c|c|c|}
\hline Convolution Layers & Output Filters & Frames per Second \\
\hline 1 & 32 & 29 \\
\hline 2 & $64-32$ & 47 \\
\hline 3 & $32-32-64$ & 80 \\
\hline 4 & $32-32-32$ & 90 \\
\hline
\end{tabular}

The CNN with 1 layer using 32 output filters has 16,590,085 parameters. The overall accuracy of this model is $\sim 99.1986 \%$. The speed of classification on the Jetson TX1 for the TensorRT model is 29 frames per second. That would give the system a granularity of $g=$ $1.8 / 29=\sim 0.062 \mathrm{~m}=\sim 6.2 \mathrm{~cm}$, using Equation (1).

The CNN with 2 layers using the 64-32 combination of output filters has 3,985,573 parameters. The overall accuracy of this model is $\sim 98.4862 \%$. The speed of classification on the Jetson TX1 for the TensorRT model is 47 frames per second. That would give the system a granularity of $g=1.8 / 47=\sim 0.038 \mathrm{~m}=\sim 3.8 \mathrm{~cm}$, using Equation (1).

The CNN with 3 layers using the 32-32-64 combination of output filters has 1,835,365 parameters. The overall accuracy of this model is $\sim 99.6438 \%$. The speed of classification on the Jetson TX1 for the TensorRT model is 80 frames per second. That would give the system a granularity of $g=1.8 / 80=\sim 0.023 \mathrm{~m}=\sim 2.3 \mathrm{~cm}$, using Equation (1).

The CNN with 3 layers using the 32-32-32 combination of output filters has 922,949 parameters. The overall accuracy of this model is $\sim 97.2395 \%$. The speed of classification on the Jetson TX1 for the TensorRT model is 90 frames per second. That would give the system a granularity of $g=1.8 / 90=\sim 0.02 \mathrm{~m}=\sim 2 \mathrm{~cm}$, using Equation (1). 
When we compare the 3 layer $\mathrm{CNN}$ using the 32-32-64 combination of output filters with the 3 layer CNN using the 32-32-32 combination of output filters, we get the following results. The granularity difference is $0.3 \mathrm{~cm}$, and an overall accuracy difference is $\sim 2.4043 \%$. The speed of classification should not be chosen over this difference in accuracy. Therefore, the best performing $\mathrm{CNN}$ is the 3 layers $\mathrm{CNN}$ with the 32-32-64 combination of output filters. 


\section{Conclusion}

In this thesis, a non-invasive control system is proposed for controlling an electric wheelchair. The control task was performed by interoperating the facial gestures of the users into five classes of commands. Sixteen convolutional neural networks have been experimented with and used to classify the facial gestures. The experimental results show that the 3 layer CNN using the 32-32-64 combination of output filters achieves the best results.

This system can make life easier for people with physical limitations. By taking a few pictures of the user and training with them on proposed CNN, the system is highly customizable. It can work on any given tasks, e.g., use commands to call for help. It is lightweight and does not require much power to operate.

The speed of classification is more than what is required for the embedded system, which can only grab 30 frames per second. Therefore, in terms of speed and accuracy, the CNN with 3 layers using the 32-32-64 combination of output filters has proven optimal for this system.

\subsection{Future Work}

For future work, the GPIOs need to be connected to a DC motor controller, e.g., on a wheelchair. It will first be tested on a RC car for POC. Additionally, for performing realtime experiments from video input, which should be the system's end goal, the image classification code needs to be adapted to use video as input.

The CNN currently uses three channel images; changing to grayscale images would be a logical step for more experimentation. The onboard camera module can be set to grayscale image grabbing. It would be interesting to compare results using grayscale images with results from this thesis.

Other camera modules should be tested for grabbing more frames per second. That would increase the granularity of the proposed system capabilities. The granularity is $6 \mathrm{~cm}$ with the current onboard camera module. The granularity can be $2.3 \mathrm{~cm}$ with another camera module.

For safety purposes, a fail-safe needs to be added to the system to make it safer. An example would be voice commands for initiating and terminating the workflow of the proposed control system. If no command is registered three times in a row, the system needs to do something to prevent accidents. For the user to not drive into things, there needs to be an obstacle avoidance control system in conjunction with the proposed control system, such as ultrasonic sensors with a novel algorithm that overrides the Gesture Interpretation control system. 



\section{References}

[1] J. Kim et al., "Assessment of the Tongue-Drive System Using a Computer , a Smartphone, and a Powered-Wheelchair by People With Tetraplegia," IEEE Trans. Neural Syst. Rehabil. Eng., vol. 24, no. 1, pp. 68-78, 2016.

[2] R. L. Madarasz, L. C. Heiny, R. F. Cromp, and N. M. Mazur, "The Design of an Autonomous Vehicle for the Disabled," IEEE J. Robot. Autom., vol. 2, no. 3, pp. 117126, 1986.

[3] S. P. Levine, D. A. Bell, L. A. Jaros, R. C. Simpson, Y. Koren, and J. Borenstein, "The NavChair Assistive Wheelchair Navigation System," IEEE Trans. Rehabil. Eng., vol. 7, no. 4, pp. 443-451, 1999.

[4] P. Majaranta and A. Bulling, "Eye Tracking and Eye-Based Human--Computer Interaction," in Advances in Physiological Computing, S. H. Fairclough and K. Gilleade, Eds. London: Springer London, 2014, pp. 39-65.

[5] M. A. Eid, N. Giakoumidis, and A. El Saddik, "A Novel Eye-Gaze-Controlled Wheelchair System for Navigating Unknown Environments: Case Study with a Person with ALS," IEEE Access, vol. 4, pp. 558-573, 2016.

[6] F. Ben Taher, N. Ben Amor, and M. Jallouli, "A multimodal wheelchair control system based on EEG signals and Eye tracking fusion," INISTA 2015 - 2015 Int. Symp. Innov. Intell. Syst. Appl. Proc., pp. 1-8, 2015.

[7] P. Viola and M. J. Jones, "Robust Real-Time Face Detection," Int. J. Comput. Vis., vol. 57, no. 2, pp. 137-154, May 2004.

[8] J. W. Machangpa and T. S. Chingtham, "Head Gesture Controlled Wheelchair for Quadriplegic Patients," Procedia Comput. Sci., vol. 132, no. Iccids, pp. 342-351, 2018.

[9] J. Lu, T.-H. Chan, Z. Zeng, Y. Ma, S. Gao, and K. Jia, "PCANet: A Simple Deep Learning Baseline for Image Classification?," IEEE Trans. Image Process., vol. 24, no. 12, pp. 5017-5032, 2015.

[10] Y. Taigman, M. Yang, M. Ranzato, and L. Wolf, "DeepFace: Closing the gap to human-level performance in face verification," Proc. IEEE Comput. Soc. Conf. Comput. Vis. Pattern Recognit., pp. 1701-1708, 2014.

[11] M. Arsenovic, S. Sladojevic, A. Anderla, and D. Stefanovic, "FaceTime - Deep 
learning based face recognition attendance system," SISY 2017 - IEEE 15th Int. Symp. Intell. Syst. Informatics, Proc., pp. 53-57, 2017.

[12] H. Li, Z. Lin, X. Shen, J. Brandt, and G. Hua, "A convolutional neural network cascade for face detection," Proc. IEEE Comput. Soc. Conf. Comput. Vis. Pattern Recognit., vol. 07-12-June-2015, pp. 5325-5334, 2015.

[13] W. J. Baddar, J. Son, D. H. Kim, S. T. Kim, and Y. M. Ro, "A deep facial landmarks detection with facial contour and facial components constraint," Proc. - Int. Conf. Image Process. ICIP, vol. 2016-Augus, pp. 3209-3213, 2016.

[14] Y. Sun, X. Wang, and X. Tang, "Deep Learning Face Representation by Joint Identification-Verification,” pp. 1-9, 2014.

[15] Y. Sun, X. Wang, and X. Tang, "Deeply learned face representations are sparse, selective, and robust," Proc. IEEE Comput. Soc. Conf. Comput. Vis. Pattern Recognit., vol. 07-12-June, pp. 2892-2900, 2015.

[16] “Jetson TX1 Module | NVIDIA Developer." [Online]. Available: https://developer.nvidia.com/embedded/jetson-tx1. [Accessed: 10-Oct-2019].

[17] "Pascal GPU Architecture | NVIDIA." [Online]. Available: https://www.nvidia.com/en-us/data-center/pascal-gpu-architecture/. [Accessed: 24Apr-2019].

[18] K. Zhang, Z. Zhang, Z. Li, and Y. Qiao, "Joint Face Detection and Alignment Using Multitask Cascaded Convolutional Networks," IEEE Signal Process. Lett., vol. 23, no. 10, pp. 1499-1503, 2016.

[19] "An intuitive guide to Convolutional Neural Networks." [Online]. Available: https://www.freecodecamp.org/news/an-intuitive-guide-to-convolutional-neuralnetworks-260c2de0a050/. [Accessed: 21-Nov-2019].

[20] “A Gentle Introduction to the Rectified Linear Unit (ReLU)." [Online]. Available: https://machinelearningmastery.com/rectified-linear-activation-function-for-deeplearning-neural-networks/. [Accessed: 21-Nov-2019].

[21] "Understanding Convolutional Neural Networks for NLP - WildML." [Online]. Available: http://www.wildml.com/2015/11/understanding-convolutional-neuralnetworks-for-nlp/. [Accessed: 21-Nov-2019].

[22] "Convolutional Neural Networks (CNN): Step 3 - Flattening - Blogs SuperDataScience - Big Data | Analytics Careers | Mentors | Success." [Online]. Available: https://www.superdatascience.com/blogs/convolutional-neural-networkscnn-step-3-flattening. [Accessed: 21-Nov-2019]. 
[23] "Layers - Slugnet 0.0 .1 documentation." [Online]. Available: https://slugnet.jarrodkahn.com/layers.html. [Accessed: 21-Nov-2019].

[24] “TikZ/Dropout at master · PetarV-/TikZ · GitHub." [Online]. Available: https://github.com/PetarV-/TikZ/tree/master/Dropout. [Accessed: 21-Nov-2019]. 



\section{Appendix A}

\section{Code for Training CNN 1}

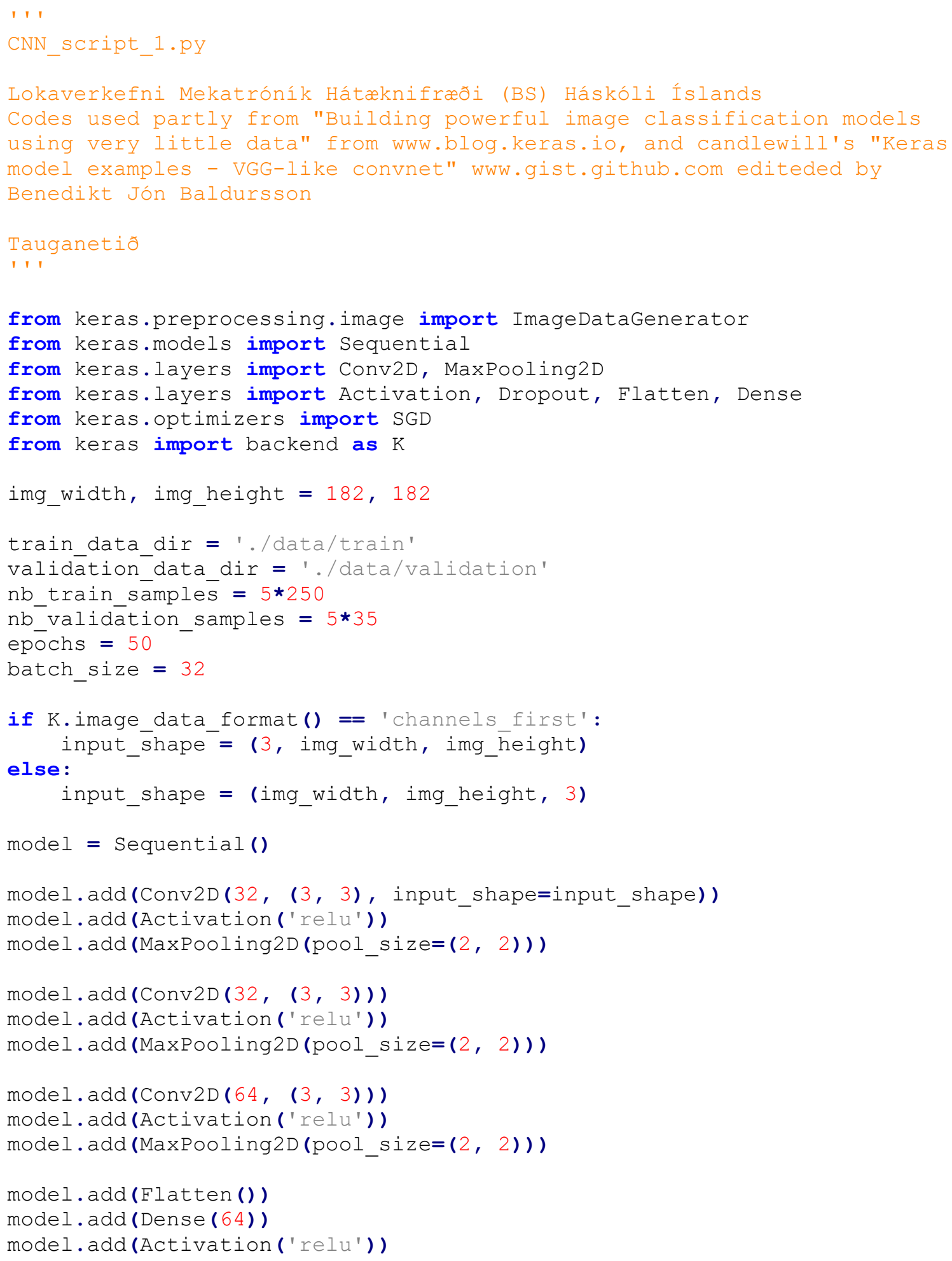




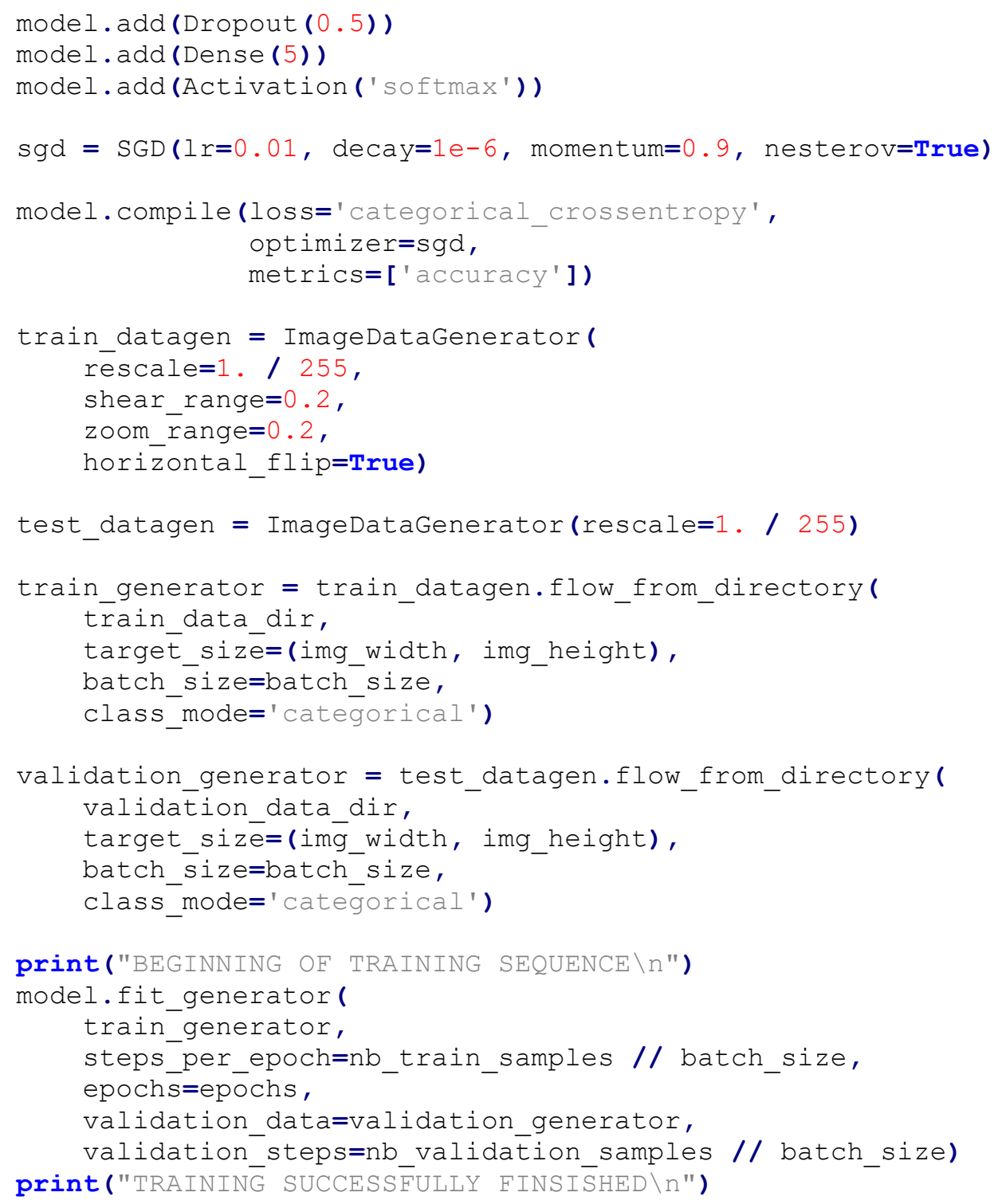




\section{Keras to TensorRT Conversion Code}

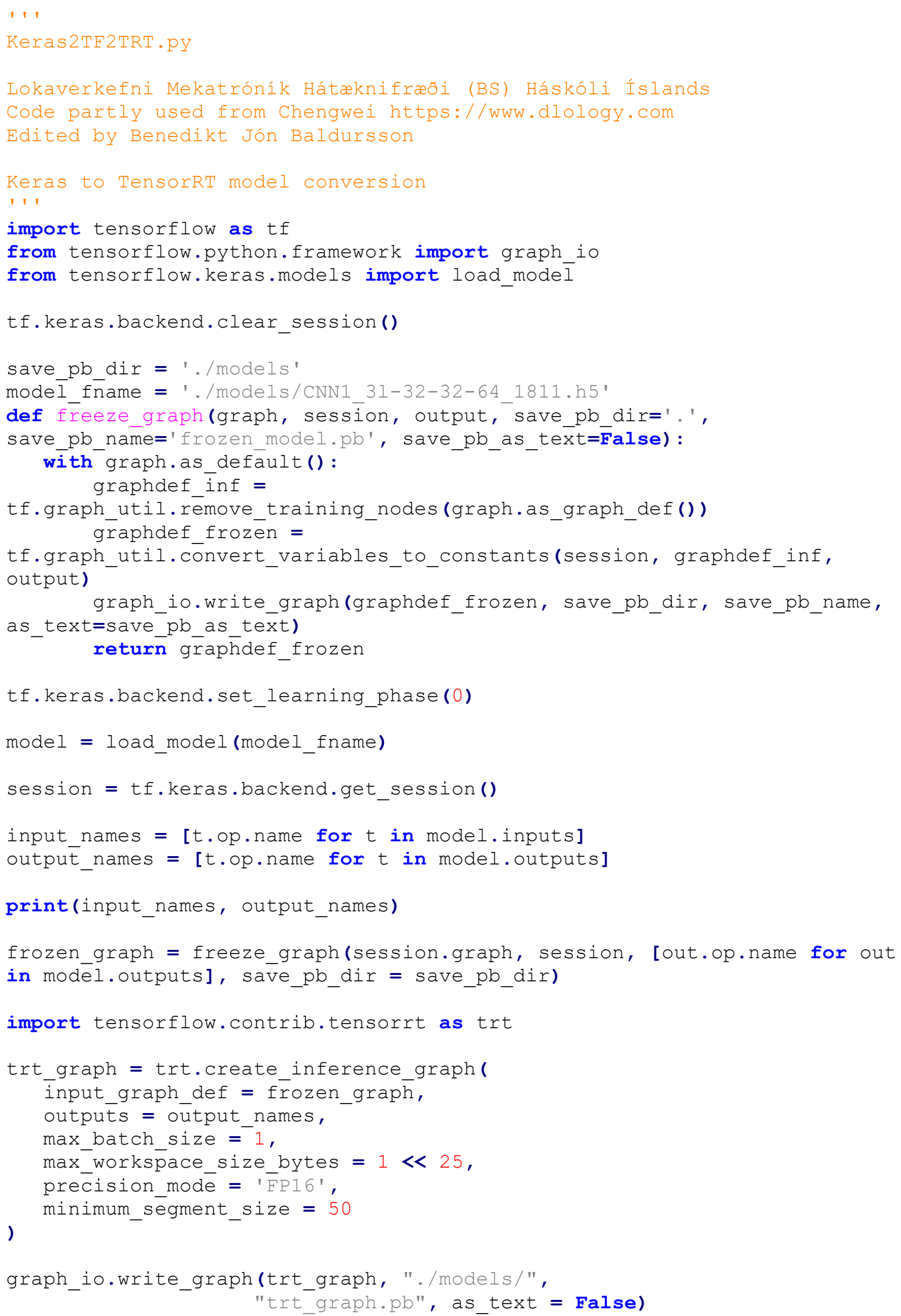





\section{Appendix B}

1. Sample of Expression Variance in Dataset

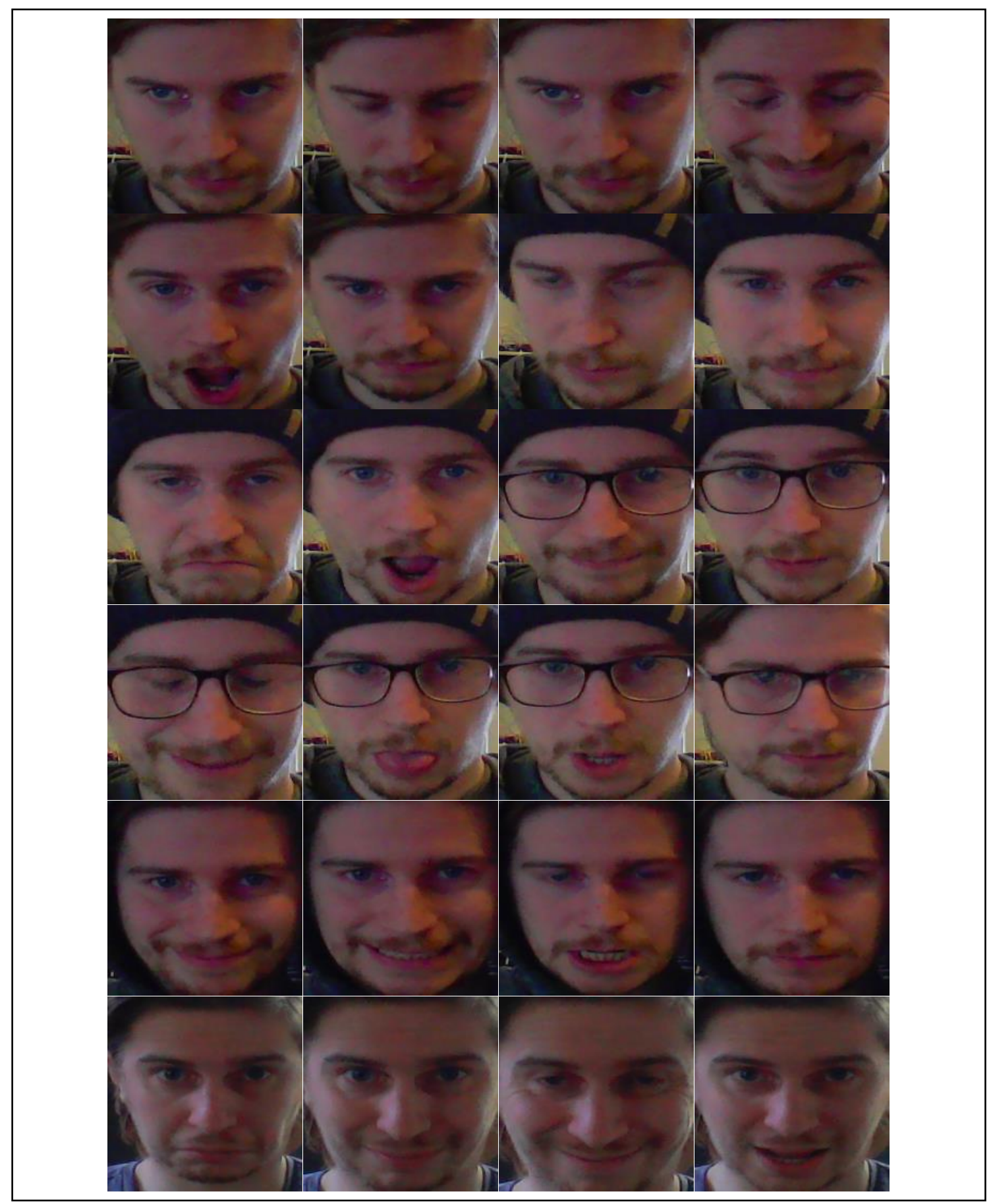

Figure 15. Sample of different expressions in dataset. 


\section{Misclassified Images - CNN 1}

a. Misclassifications in Stop Class

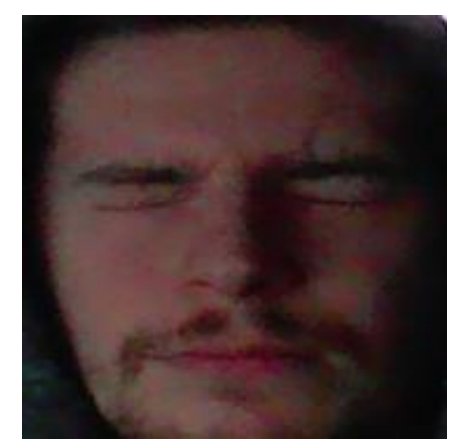

Figure 16. Stop misclassified as Forward.

Image from Stop class in the test dataset, Figure 16, was misclassified as Forward.

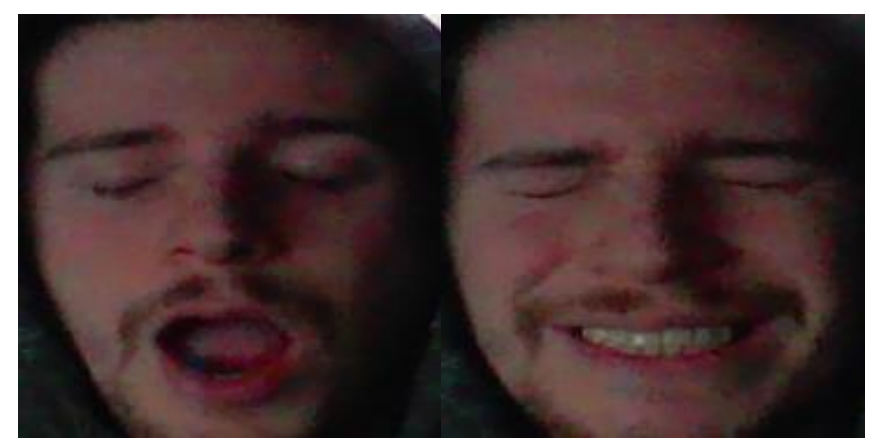

Figure 17. Stop misclassified as Backward.

Image from Stop class in the test dataset, Figure 17, was misclassified as Backward.

b. Misclassifications in Left Class

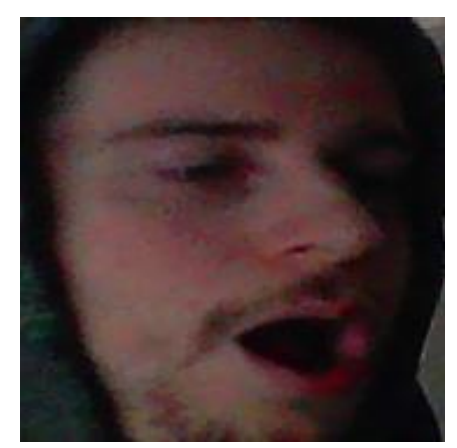

Figure 18. Left misclassified as Forward.

Image from Left class in the test dataset, Figure 18, was misclassified as Forward. 